\title{
Optimal Flying Wings: A Numerical Optimization Study
}

\author{
Charles A. Mader* \\ University of Toronto Institute for Aerospace Studies \\ Toronto, Ontario, Canada \\ Joaquim R.R.A. Martins ${ }^{\dagger}$ \\ Department of Aerospace Engineering, University of Michigan \\ Ann Arbor, Michigan
}

\begin{abstract}
The optimal shape of flying wings for subsonic and transonic speeds is examined using high-fidelity numerical optimization tools. The first result in the study is a lift-constrained drag minimization, performed on an un-swept, rectangular wing. By varying the spanwise twist distribution of the wing we are able to reproduce the elliptic optimum predicted by low speed inviscid theory. Using this result as a reference, we explore four different optimization formulations, considering the addition of bending moment constraints, static stability constraints and dynamic stability constraints. In each case, we explore the design space of the problem using both planform and surface shape variables to determine the optimal shape. Using these techniques, we show that the addition of stability constraints has a significant impact on the optimal surface shape of the wing. In particular, we show that at lower speeds, airfoil shape is sufficient to satisfy static stability constraints, while dynamic stability constraints require the addition of sweep. We also show that at higher speeds, shape is insufficient to satisfy either stability constraint, static or dynamic, and that the addition of sweep is necessary.
\end{abstract}

\section{Introduction}

The design of flying wing aircraft is a complex, coupled problem. In addition to the strong aerostructural coupling seen in typical aircraft design, there is a strong coupling between the aerodynamic efficiency of the outer mold line (OML) and the trim and stability of the aircraft. This strong coupling requires a delicate balance between optimizing the aircraft for aerodynamic performance and maintaining the necessary stability characteristics of the aircraft [1]. Thus, multidisciplinary design optimization (MDO) is an invaluable tool in flying wing design. While stability characteristics have been considered in aircraft MDO using lower fidelity tools [2, 3], as efficiency standards are increased, more accurate, higher fidelity analyses will be required in the design process to make the most of new configurations. Further, while high-fidelity aerodynamic optimization has matured significantly over the last 20 years $[4,5,6,7]$, the ability to consider stability characteristics using the same high-fidelity aerodynamic information has not been incorporated in these studies. In this work, we examine a series of increasingly complex optimization problems to study the effects that various structural and stability constraints have on the optimal design. In particular, we examine the trade-offs between optimal aerodynamic performance and the limits imposed by structural and stability constraints. The consideration of stability is enabled by the recently developed time-spectral stability derivative method [8], which efficiently computes stability derivatives, and their sensitivities, using computational fluid dynamics (CFD).

The main body of this study is arranged in five sections. Section II provides a brief overview of the tools and methods used in the study, while providing references to other work with more details on each method or tool. Section III then provides a review of MDO methods with particular emphasis on the techniques used in this work. Section IV introduces the design problem and discusses the various optimization formulations that are used in the study. Finally, Sections V and VI present the results of the study, first examining the qualitative trends of the solutions, then following up with a discussion of the precise numerical results obtained in each case.

\section{Methodology}

The methodologies used to conduct this study are driven by two main factors: the desire to consider transonic aircraft, and the desire to consider stability constraints. The first consideration necessitates, at a minimum, the solution of the Euler equations using CFD. The second requires the computation of the stability characteristics of the aircraft

\footnotetext{
*Ph.D Candidate, AIAA Student Member

$\dagger$ Associate Professor, AIAA Senior Member
} 
along with their sensitivities with respect to the design variables of interest. In this section, we give a brief overview of the techniques and methods used to conduct this study, while referring the reader to references for more details.

\section{A. Euler CFD}

The core module of the following study is a high-fidelity CFD solver. The tool used in this particular case is SUmb [9], which can solve the Euler equations as well as the fully turbulent Reynolds-averaged Navier-Stokes (RANS) equations. For this study we have limited ourselves to the steady and time-spectral Euler equations. In this context, with a moving-grid formulation included for use with the time-spectral equations, the governing equations can be written as:

$$
\frac{\partial \zeta}{\partial t}+\frac{\partial f_{i}}{\partial y_{i}}=0,
$$

where $y_{i}$ are the coordinates in the $i^{\text {th }}$ direction. The states and fluxes can then be written as:

$$
\zeta=\left[\begin{array}{c}
\rho \\
\rho u_{1} \\
\rho u_{2} \\
\rho u_{3} \\
\rho e_{t}
\end{array}\right], \quad f_{i}=\left[\begin{array}{c}
\rho u_{i}-\rho w_{i} \\
\rho u_{i} u_{1}-\rho w_{i} u_{1}+p \delta_{i 1} \\
\rho u_{i} u_{2}-\rho w_{i} u_{2}+p \delta_{i 2} \\
\rho u_{i} u_{3}-\rho w_{i} u_{3}+p \delta_{i 3} \\
\rho u_{i}\left(e_{t}+p\right)-\rho w_{i} e_{t}
\end{array}\right],
$$

where $w$ represents the velocity of the grid. Mader and Martins [10] provide a detailed derivation of this formulation as applied to SUmb.

For the steady cases we write Equation (1) as:

$$
\frac{\partial \zeta}{\partial t}+R(\zeta)=0
$$

and solve for $R(\zeta)=0$.

For the time-spectral case, we start with Equation (3) and modify the time derivative to be the spectral operator derived by Gopinath [11], yielding:

$$
R_{T S}(\zeta)=D_{t} \zeta^{n}+R\left(\zeta^{n}\right)=0,
$$

$D_{t}$ is a spectral operator that spans all $N$ time instances in the solution and $n$ represents each of those $N$ time instances. By solving the $N$ coupled time instances, we obtain a coupled set of solutions that represent the periodic, steady-state solution to the given time-spectral problem.

The solution of either Equation (3) or (4), produces a field solution of the aerodynamic states from which the necessary values of lift, drag and moment can be calculated. Further, as described in Section F, the time-spectral solution of Equation (4) also provides the information necessary to evaluate certain stability derivatives that are needed for the constraints described in Section G.

\section{B. MDO Framework}

In addition to the flow solver, which is the main tool used in this work, there are a number of other tools that are necessary to complete the presented optimizations. These include tools for optimization, geometry handling, mesh handling and a variety of simple auxiliary analysis necessary for the computation of the various constraints. In the following section, we briefly describe each of these tools.

\section{Optimization Algorithm}

The optimization tool used in this work is pyopt [12], a Python based optimization framework that allows the user to access, through a common interface, a variety of numerical optimization packages. Because the cost of a single CFD solution is relatively high, we have chosen to use gradient-based optimization techniques for this study. pyOpt provides interfaces to a variety of gradient-based optimizers, both open source and licensed. In this work, we have chosen to use the pyOpt interface to SNOPT [13], which is an SQP based optimizer, because SNOPT is proficient at handling large nonlinear optimization problems. 


\section{Geometry Modelling}

The geometry modelling for this work is conducted by a series of layers. The top layer is a conceptual design description based on simple planform variables. This level of the geometry is handled by PYACDT [14], a Python based, object-oriented aircraft design tool. This tool models the planform of the aircraft and provides information for the conceptual level mass and inertia computations used in the computation of the stability constraints.

The high-fidelity geometry representation is modeled by PYPSG [15]. This tool is used to create a water-tight surface representation of the aircraft, a set of spline volumes enveloping the aircraft, and a set of reference axes inside the aircraft. The set of volumes enveloping the aircraft are used in conjunction with a free form deformation (FFD) technique [15] to handle the detailed geometry manipulations during the optimization. The surface points on the CFD mesh are embedded parametrically in these spline volumes, such that when the volumes are moved or deformed, the CFD surface mesh is modified as well. The reference axes are used to tie together the control points of the FFD volumes enveloping the aircraft. By tying the control points to a reference axis, we are able to create the effect of physical design variables such as sweep, twist, taper, area, span and chord, which are more meaningful to a designer than the location of arbitrary control points. The geometry is structured such that the planform changes specified in the PYACDT geometry layer are transfered to the CFD surface mesh through these reference axes.

\section{Mesh Deformation}

Once the CFD surface mesh is deformed, that deformation needs to be propagated to the CFD volume mesh. This is handled using an efficient mesh deformation technique developed by Kenway et al. [15]. Using this technique, the CFD mesh is represented by a coarse finite-element mesh. This mesh is deformed using the perturbations of the coarse mesh surface nodes as input values. When the finite-element equations are solved, the large surface deformations are propagated to the remainder of the volume mesh. Then, an algebraic mesh deformation scheme, based on trans-finite interpolation, is used to propagate the surface deformations on the blocks immediately adjacent to the surface. This provides a fully updated volume mesh to the CFD solver.

\section{Geometric Constraints}

To aid in producing realistic results, two geometry constraints are included in the optimizations. The first is a set of thickness constraints, included to prevent the optimizer from making the wing unrealistically thin. These constraints are implemented by computing thicknesses at a variety of locations in the wing and constraining those values to be no smaller than the initial values. The locations where these thicknesses are evaluated are defined by the user. In this case, the thickness was constrained to maintain the thickness of the NACA 0012 profile at 1,50 and 99 percent chord at 10 evenly spaced stations along the wing.

The second geometry constraint is a constraint on the leading and trailing edge control points of the FFD volume. If these points are allowed to move freely, they can reproduce the same degrees of freedom as twist variables, producing an ill-posed optimization problem. To prevent this, the control points at each of the leading and trailing edges are constrained to move in equal and opposite directions so that the mid point of the FFD does not move with the shape variable perturbations.

\section{Center of Gravity Calculation}

The center of gravity (CG) location is fundamental for most of the calculations in this study. Firstly, it is the point about which all aerodynamic moments are calculated. Therefore, the moment coefficient, $C_{m}$, and all of its derivatives - in this case $C_{m_{\alpha}}, C_{m_{\dot{\alpha}}}$ and $C_{m_{q}}$ - are strongly dependent on the CG location. Secondly, the mass moment of inertia calculation uses the CG location as a reference point. All of these quantities are necessary for the computation of the stability parameters that are to be constrained. Therefore, having an appropriate CG location is necessary to produce meaningful design optimization results.

In this work, a relatively simple wing CG calculation is implemented. The method is derived from the work of Chai et al. [16], where the authors state that the wing CG for a normal transport wing is located between the fore and aft spars along the wing mean aerodynamic chord (MAC). Since flying wings are being examined in this study, we assume that this estimate of the wing CG is a reasonable estimate of the CG for the entire aircraft.

The starting point for the calculation is the computation of the wing MAC and the location of its quarter chord. These calculations are based on the methods presented in ESDU item 76003 [17]. One then determines the location of the intersections of the spars with the MAC. In this work, the fore and aft spars are assumed to be at $25 \%$ and $75 \%$ of the MAC, respectively. The longitudinal location of the $\mathrm{CG}$ can then be determined as a percentage $-C G_{\%}-$ of the

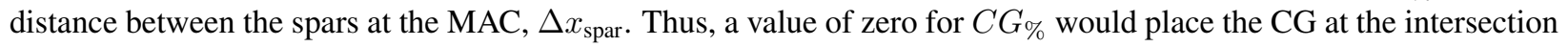


of the forward spar and the MAC, a value of one for $C G_{\%}$ would place the $\mathrm{CG}$ at the intersection of the rear spar and the MAC, and a value of negative one for $C G_{\%}$ would place the $\mathrm{CG}$ a distance of $\Delta x_{\text {spar }}$ in front of the intersection of the forward spar and the MAC. Figure 1 illustrates the key parameters in the approach.

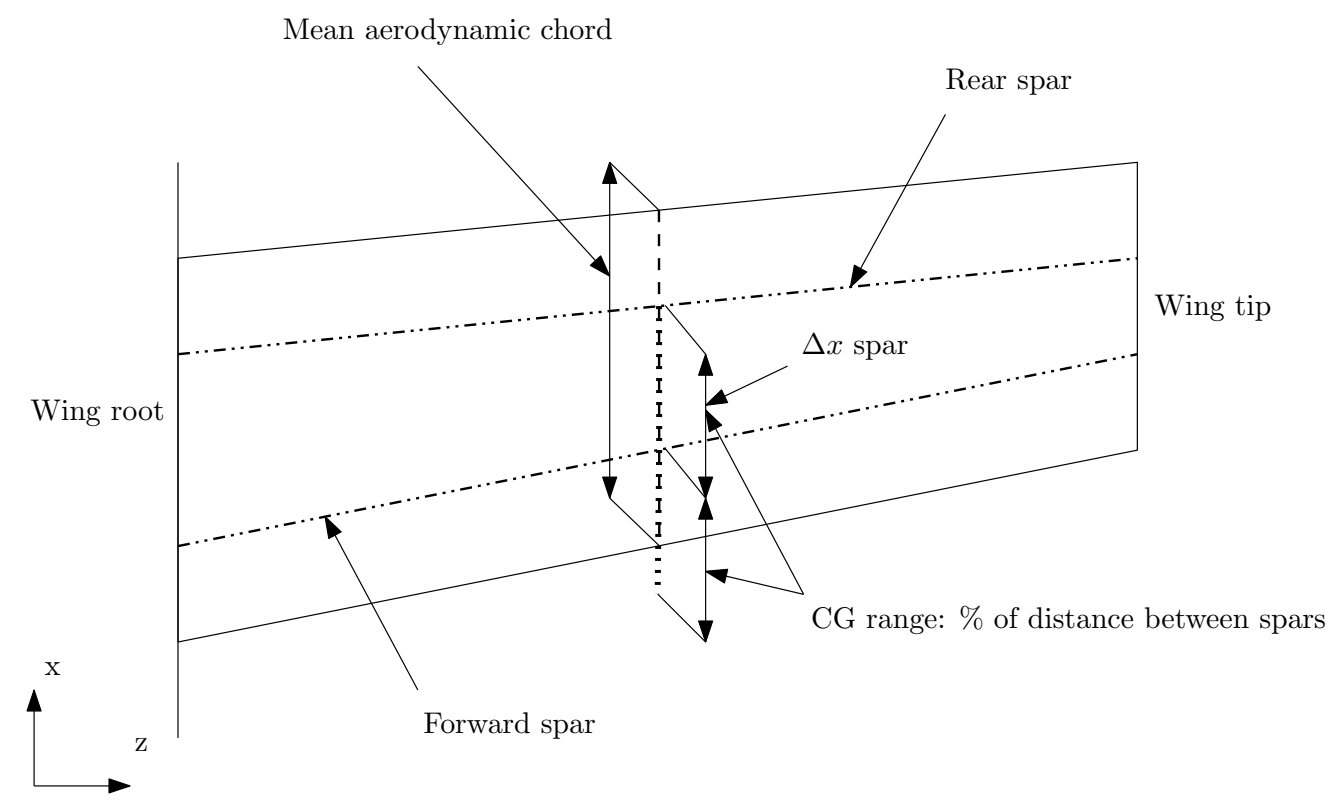

Figure 1. Diagram of CG calculation

\section{Moment of Inertia Calculations}

The moment of inertia plays a key role in the dynamic stability constraints. Just as mass relates force to acceleration, moment of inertia relates moment to rotational acceleration. As a result, the pitching moment of inertia $-I_{z z}-$ shows up in the normalization of all of the moment terms in the short-period approximation described in Section 2.

The calculations for the moments of inertia are based on first principles. The definition of moment of inertia is:

$$
I=\int_{\Omega} r^{2} \underline{m},
$$

where to compute this value, the domain of integration, $\Omega$, is set to be the surface of the aircraft. In this case, the surface is defined by a B-spline surface using our geometry modeler. Having defined the surface in this fashion, a discrete surface mesh can be created by evaluating the spline over a uniform distribution of values in $u, v$ parameter space. A sample wing mesh is shown in Figure 2. These discrete surface cells are then given a thickness $(t)$ and a density $(\rho)$, and the area of each cell is computed using a standard vector product. The area and thickness of the cell are then multiplied to get the volume of the cell, which when multiplied by the density gives the total mass for each of the discrete cells on the surface mesh. These lumped masses are illustrated as spheres in Figure 2. Considering this mass to be located at the centroid of the cell, the moment of inertia can the be computed as:

$$
I=\sum m r^{2} .
$$

In this case, $r$ is the distance from the lumped mass to the CG, perpendicular to the rotational axis of interest. Therefore, for $I_{z z}$ :

$$
r^{2}=\left(y_{\text {mass }}-y_{\mathrm{CG}}\right)^{2}+\left(x_{\text {mass }}-x_{\mathrm{CG}}\right)^{2} .
$$

and the moment of inertia of interest for the pitching calculations is:

$$
I=\sum m\left[\left(y_{\mathrm{mass}}-y_{\mathrm{CG}}\right)^{2}+\left(x_{\mathrm{mass}}-x_{\mathrm{CG}}\right)^{2}\right] .
$$

Because this value approximates the moment of inertia of only the wing skin, an additional multiplier is added to the computation to account for the remaining mass distribution in the aircraft. This parameter is given to the optimizer as a variable to allow it to satisfy the constraints in the dynamic stability constrained optimization. 


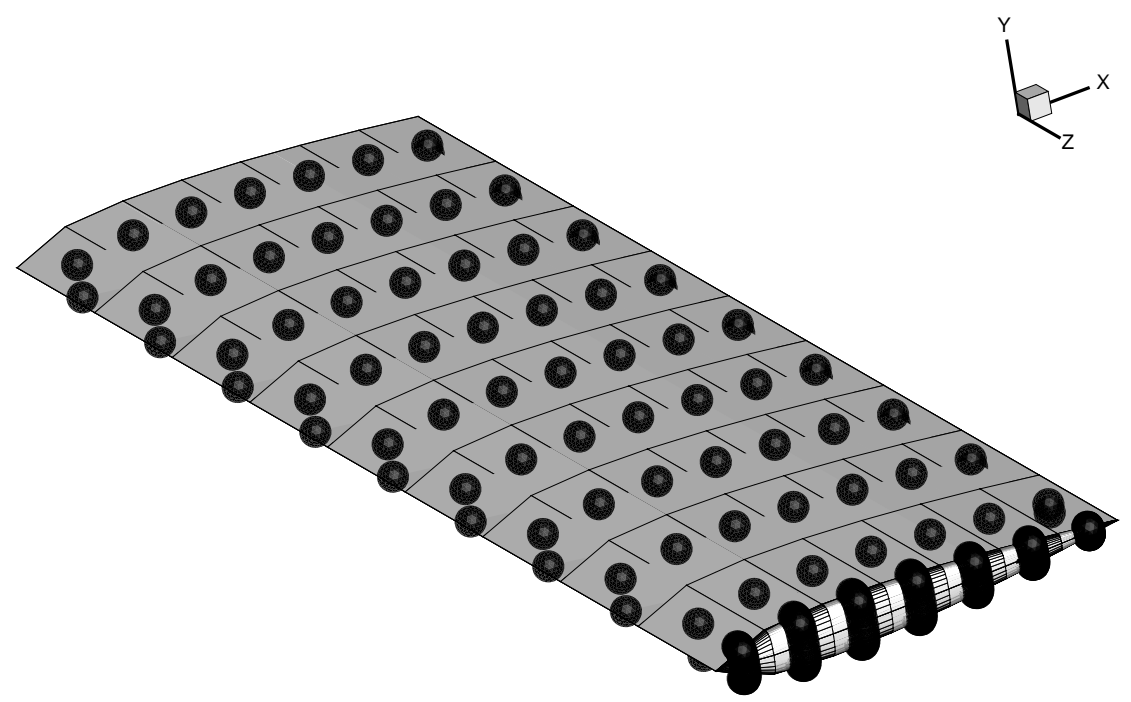

Figure 2. Discrete surface mesh with lumped masses at the cell centroids

\section{E. Root Bending Moment Constraint}

The root bending moment constraint is included to give some consideration to the structural implications of the varying wing shapes. There are well known trade-offs between aerodynamic and structural performance for wings, the most prominent of which has to do with the effects of span. Increasing the span reduces induced drag. However, for the same total lift, a span extension also increases the bending moment at the root of the wing, requiring a heavier structure to support the aerodynamic load. A similar effect is caused by wing sweep. For shearing sweep - where individual sections of the wing are translated in the flow-wise direction to sweep the wing - the effective structural length of the wing increases as the wing is swept. This can be counteracted by reducing the span of the wing, which introduces a trade-off between induced drag and wave drag at transonic Mach numbers. Therefore, if span and sweep are used as design variables, it is necessary to account for these trade-offs between aerodynamic performance and structural weight. In this work, the root bending moment is used as a proxy for the structural performance of the wing. The assumption implicit in this approach is that two wings with the same root bending moment require the same amount of material to support the load on the wing and thus have the same weight. This is a relatively simplistic assumption, but serves as a useful metric for including structural considerations in the optimization.

To compute the bending moment coefficient, the pressure is integrated over the aircraft to get the force and moment coefficients about a reference point, $x_{\text {ref }}$. This computation yields the values of $C_{f_{x}}, C_{f_{y}}, C_{f_{z}}, C_{m_{x}}, C_{m_{y}}$ and $C_{m_{z}}$, which are the force and moment coefficients in the three principal Cartesian axes. A bending reference point, $x_{\text {bend }}$, is then specified at the root of the wing. This is the point about which the net bending moment is calculated. The bending moment is then calculated as:

$$
\begin{aligned}
C_{\text {bend }_{x}} & =C_{m_{x}}+C_{f_{y}} \frac{x_{\text {bend }_{z}}-x_{\mathrm{ref}_{z}}}{\bar{c}_{\mathrm{ref}}}-C_{f_{z}} \frac{x_{\text {bend }_{y}}-x_{\mathrm{ref}_{y}}}{\bar{c}_{\mathrm{ref}}} \\
C_{\text {bend }_{z}} & =C_{m_{z}}-C_{f_{y}} \frac{x_{\text {bend }_{x}}-x_{\mathrm{ref}_{x}}}{\bar{c}_{\mathrm{ref}}}+C_{f_{x}} \frac{x_{\text {bend }_{y}}-x_{\mathrm{ref}_{y}}}{\bar{c}_{\mathrm{ref}}} \\
C_{\mathrm{b}} & =\sqrt{C_{\text {bend }_{x}}^{2}+C_{\text {bend }_{z}}^{2}}
\end{aligned}
$$

where $\bar{c}_{\text {ref }}$ is the reference chord length, $C_{\mathrm{b}}$ is the total root bending moment coefficient and $C_{\text {bend }_{x}}$ and $C_{\text {bend }_{z}}$ are the Cartesian components of the root bending moment about the bending reference point a the wing root.

A diagram illustrating the various components of this calculation is shown in Figure 3. Note that since the torsional component of the wing moment is non-zero, the effective bending moment is not necessarily aligned with the wing. Also note that as the wing is swept, this torsional component increases, causing the combined effective bending moment to sweep with the wing. Therefore, we assume that the wing can be supported normal to the effective total 
bending moment, regardless of the wing sweep and pressure distribution. Thus, only the total magnitude of the bending coefficient, $C_{\mathrm{b}}$, is considered. Note that the reference value of $C_{\mathrm{b}}$ used to constrain the wing has a significant effect

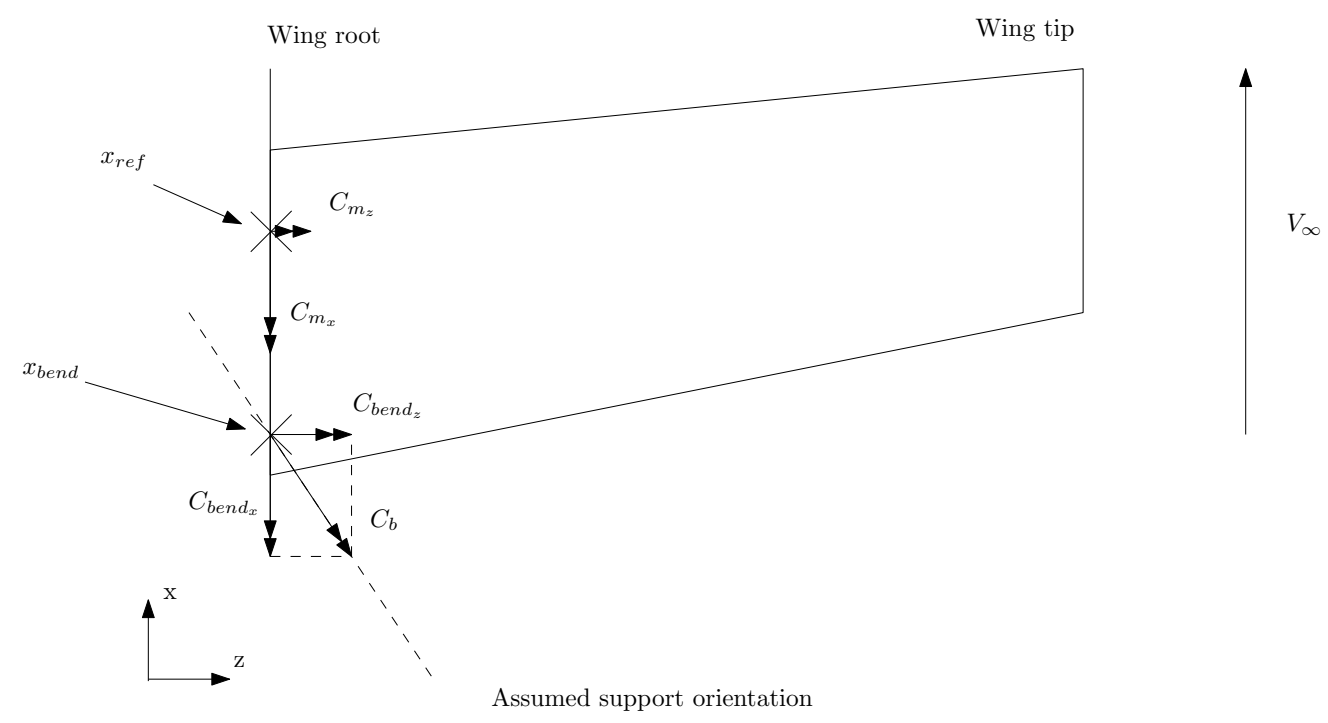

Figure 3. Bending moment calculation

on the optimal design. In this study, the value from the elliptical optimum - computed at $\mathrm{M}=0.5-$ is used as the reference value. This value is then scaled with the root cross section to ensure that as the optimizer increases the aspect ratio of the wing, the allowable bending moment is reduced to account for the reduced second moment of area at the wing root. The reasoning behind this scaling is based on the definition of bending stress,

$$
\sigma=\frac{M y}{I}
$$

Rearranging to get an expression for the moment and performing a dimensional analysis yields,

$$
M \propto \frac{\sigma L^{4}}{L}=\sigma L^{3} .
$$

Note that because we are assuming a constant $t / c$ ratio, both the thickness and chord dimensions scale with $L$. Expressing the moment in terms of coefficients, we get

$$
q A \bar{c} C_{\text {bend }} \propto \sigma L^{3}
$$

Also, $A$ is constrained to be constant in the optimizations, therefore

$$
C_{\text {bend }} \propto \frac{\sigma L^{3}}{q A L}
$$

or,

$$
C_{\text {bend }} \propto \frac{\sigma L^{2}}{q A}
$$

Thus, for constant $q A$, the allowable bending moment must be scaled with $L^{2}$ to enforce a constant allowable bending stress at the wing root. Note that in this specific case, $L$ is the ratio between the optimized root chord and the initial root chord of $1 \mathrm{~m}$.

\section{F. Stability Derivatives}

The stability constraints used in this work are based on linear flight dynamic theory. This theory uses aircraft stability derivatives to represent aerodynamic characteristics of the aircraft in the model. The aircraft stability derivatives are calculated using the time-spectral stability derivative formulation developed by Mader and Martins [8]. This approach is similar to the methods presented by Murman [18] and DaRonche et al. [19] and is motivated by the complex number $\dot{\alpha}$ derivative methodology outlined by Etkin [20]. The approach used is essentially a forced oscillation technique. A 
time-spectral CFD solver is used to generate the solution for a prescribed oscillatory motion. This periodic solution is then used, with a linear regression technique, to generate estimates for the functionals of interest - the force or moment coefficient, the derivative of that coefficient with respect to the oscillating parameter, and the derivative of that coefficient with respect to the time derivative of the oscillating parameter. We are interested in the derivatives of lift, drag and pitch moment coefficients, $\left(C_{L}, C_{D}, C_{m}\right)$ with respect to $\alpha, q$ and their time derivatives, $\dot{\alpha}, \dot{q}$. The simple algebraic nature of this method allows it to be used in conjunction with an adjoint method to efficiently compute the gradients necessary for optimization.

\section{G. Stability Constraints}

One of the main contributions of this work is the development of a methodology that allows for the inclusion of CFD-based stability information, in aerodynamic shape optimization. The following section outlines how the stability derivatives from Section F, and the resulting linear flight dynamics model are used to formulate stability constraints for the optimization problem. Two stability constraints are considered: one based on static stability and one based on dynamic stability. A discussion of other stability criteria applied to a flying-wing configuration can be found in Agenbag [21].

\section{Static Longitudinal Stability}

To develop a static stability constraint, we turn to the definition of static margin. First, consider the definition of the moment coefficient,

$$
C_{m_{\mathrm{CG}}}=C_{m_{\mathrm{NP}}}+\left(h_{\mathrm{CG}}-h_{\mathrm{NP}}\right) C_{L}
$$

Where $h_{\mathrm{CG}}$ and $h_{\mathrm{NP}}$ are the streamwise locations of the CG and neutral point respectively, normalized by the MAC. Differentiating with respect to $\alpha$ yields,

$$
\frac{\partial C_{m_{\mathrm{CG}}}}{\partial \alpha}=\left(h_{\mathrm{CG}}-h_{\mathrm{NP}}\right) \frac{\partial C_{L}}{\partial \alpha}
$$

or, using stability derivative notation,

$$
C_{m_{\alpha}}=\left(h_{\mathrm{CG}}-h_{\mathrm{NP}}\right) C_{L_{\alpha}}
$$

Defining the static margin, $K_{n}$, as the distance between the CG and the neutral point normalized by the MAC,

$$
K_{n}=h_{\mathrm{NP}}-h_{\mathrm{CG}}
$$

and substituting this relationship back into Equation (17) results in,

$$
C_{m_{\alpha}}=-K_{n} C_{L_{\alpha}}
$$

which can be rearranged as,

$$
K_{n}=-\frac{C_{m_{\alpha}}}{C_{L_{\alpha}}} .
$$

Thus, by specifying a static margin, we can determine a meaningful value for $C_{m_{\alpha}}$.

Up to this point, the discussion has been completely general and applicable to any aircraft. By examining Equations (15) and (17), we can gain insight as to how the various stablility conditions may be satisfied for a the particular case of a flying wing. Equation (15) shows that the moment coefficient is dependent on the moment about the neutral point - which does not change with angle of attack - and the location of the neutral point with respect to the CG. Thus, the optimizer can trim the aircraft by altering three parameters: the moment, the neutral point position or the CG position. The moment coefficient about the neutral point is altered either with airfoil shape - adding reflex to the airfoil — or by twisting down the wing tips on a swept wing. The neutral point of the aircraft is altered primarily by changing the sweep of the wing. Finally, the CG location of the aircraft can be altered by changing the sweep of the wing, as well as by shifting the payload location. However, Equation (17) shows that the neutral point and CG locations both impact $C_{m_{\alpha}}$ and hence the static margin. Thus, the optimizer must simultaniously adjust the wing sweep and shape, as well as the payload location to trim the aircraft and create an acceptable static margin. These various elements are discussed along with the results in Section V. 


\section{Short Period Approximation}

As a precursor to the discussion on the dynamic stability constraint, we introduce the short period approximation to the standard, linear flight dynamic model. This approximation assumes that the variation in forward velocity $(u)$ is negligible and that the short period characteristics of the aircraft can be modeled by changes in pitch rate $(q)$ and angle of attack $(\alpha)$ alone. In this case, vertical velocity $(w)$ is used in place of the angle of attack where:

$$
\tan \alpha=\frac{w}{u} .
$$

To begin, we define a simplified version of the flight dynamic model based on the degrees of freedom described above. This gives:

$$
\left[\begin{array}{c}
\Delta \dot{w} \\
\Delta \dot{q}
\end{array}\right]=\left[\begin{array}{cc}
\frac{-Z_{w}}{m} & -u_{0} \\
\frac{-M_{\dot{w}} Z_{w}}{I_{y} m}-\frac{M_{w}}{I_{y}} & \frac{-M_{\dot{w}} u_{0}}{I_{y}}-\frac{M_{q}}{I_{y}}
\end{array}\right]\left[\begin{array}{c}
\Delta w \\
\Delta q
\end{array}\right]+\left[\begin{array}{c}
Z_{\text {control }} \\
M_{\text {control }}
\end{array}\right]\left[\Delta_{\text {control }} \cdot\right]
$$

Ultimately, as we show later in this section, the handling qualities analyses we are interested in require the frequency and damping ratio of the aircraft. These values can be determined from the characteristic equation of the $2 \times 2$ system in Equation (22). As shown in McRuer et al. [22] the relevant characteristic equation is,

$$
s^{2}+\left(\hat{M}_{\dot{w}} u_{0}+\hat{M}_{q}+\hat{Z}_{w}\right)+\hat{Z}_{w} \hat{M}_{q}-\hat{M}_{w} u_{o} .
$$

Comparing this to the typical second order characteristic equation:

$$
s^{2}+2 \zeta \omega s+q^{2}
$$

yields:

and

$$
\omega=\sqrt{\hat{Z}_{w} \hat{M}_{q}-\hat{M}_{w} u_{o}}
$$

$$
2 \zeta \omega=\left(\hat{M}_{\dot{w}} u_{0}+\hat{M}_{q}+\hat{Z}_{w}\right)
$$

or

$$
\zeta=\frac{\hat{M}_{\dot{w}} u_{0}+\hat{M}_{q}+\hat{Z}_{w}}{2 \omega} .
$$

We can now use the frequency and damping ratio of the short-period mode to evaluate the Control Anticipation Parameter, which is used as the dynamic stability constraint in this study.

\section{Control Anticipation Parameter}

The control anticipation parameter (CAP) is a method used to quantify the handling qualities of an aircraft based on its short-period characteristics. The fundamental idea behind this approach is that a pilot's ability to fly an aircraft precisely along a given flight path is related to the pilot's ability to anticipate response of the aircraft. In this approach, Bihrle [23] relates the pilots ability to anticipate the aircraft's response to a ratio between the instantaneous pitch acceleration of the aircraft and the steady state normal acceleration of the aircraft, which can be expressed as:

$$
\mathrm{CAP}=\frac{\dot{q}}{\Delta n}
$$

However, these quantities are not necessarily simple to evaluate for an aircraft. Therefore, with a little rearranging, Bihrle [23] provides the following expression:

$$
\mathrm{CAP}=\frac{\omega_{n}^{2}}{n_{\alpha}}
$$

where $n_{\alpha}$ is simply:

$$
n_{\alpha}=\frac{\frac{1}{2} \rho V^{2} S_{\text {ref }} C_{L_{\alpha}}}{g}
$$

We can now use the stability derivative methods and short-period approximation described earlier to compute the necessary values.

The United States Military has specified acceptable limits for CAP and damping ratio for various combinations of aircraft and flight conditions [24]. For an aircraft at cruise (Category B), those limits are shown in Table 1. 
Table 1. MIL-F-8785c handling qualities limits

\begin{tabular}{rrr|rr}
\hline \hline & \multicolumn{2}{c}{ Damping Ratio Limits } & \multicolumn{2}{c}{ CAP Limits } \\
\hline Lower Bound & Upper Bound & Lower Bound & Upper Bound \\
\hline Level 2 & 0.30 & 2.0 & 0.085 & 3.6 \\
Level 3 & 0.20 & 2.0 & 0.038 & 10.0 \\
\hline \hline
\end{tabular}

\section{Multidisciplinary Optimization Overview}

In order to better understand the optimization problems presented in this work and the choice of variables used herein, it is necessary to provide a brief overview of multidisciplinary design optimization (MDO) architectures. Generally speaking, an MDO architecture is the method used to handle the coupling between disciplines in a multidisciplinary optimization problem. Numerous architectures have been developed to handle this coupling and the various methods can be divided into two broad categories: monolithic architectures and decompositional architectures. Monolithic architectures — such as the multidisciplinary feasible (MDF) architecture [25], the individual discipline feasible (IDF) architecture [25] and the simultaneous analysis and design (SAND) [26] architecture - set up the problem as a single level optimization and handle the objective function optimization and interdisciplinary coupling in one optimization problem. Decompositional architectures — such as collaborative optimization (CO) [27], concurrent subspace optimization (CSSO) [28], bi-level integrated system synthesis (BLISS) [29] and analytical target cascading [30] - decompose the optimization into multiple optimization problems where each secondary optimization problem handles a portion of the overall optimization and a global coordination problem ensures that the various secondary optimization problems produce the correct multidisciplinary optimum.

Note that all of the optimization diagrams that follow, including the MDF and IDF diagrams in this section, are presented in the extended design structure matrix (XDSM) format of Lambe and Martins [31]. These diagrams are designed to show the data connectivity between the disciplines as well as the process flow of the solution algorithm. The large blocks on the diagonal represent the major analyses in the optimization process. These include the discipline analyses and the evaluation of any constraints from the converged disciplines, as well as the optimization iterations themselves. The off-diagonal nodes represent data connectivity between the disciplines, with the boxes above the diagonal representing feed-forward connections and the boxes below the diagonal representing feed-back connections. The thick gray lines represent data flow paths between the various disciplines, and the thin black line represents the process flow through the solution algorithm, which follow in numerical order. The case specific diagrams included with each problem formulation have been updated to include the actual variables used in each optimization formulation.

Returning to the discussion of architectures, two MDO architectures are relevant to this work: the MDF architecture and the IDF architecture. In the MDF architecture, shown in Figure 4, all of the disciplines are tightly coupled. Each discipline passes the relevant portion of its solution - the coupling variables, $y$ - to the other disciplines directly. This coupled system is then iterated to convergence at each optimization iteration before taking a step in the design space. As a result of this tight coupling, the MDF approach also requires a complete, coupled-sensitivity analysis of all the disciplines.

In the IDF architecture, shown in Figure 5, the disciplines are completely decoupled. Each discipline is solved once per optimization iteration and is responsible for its own sensitivities. In this case, the optimizer passes target coupling variables, $y^{t}$, to each discipline to facilitate the solution of the individual disciplines. To ensure that the system is multidisciplinary feasible at the optimum solution, an additional set of compatibility constraints, $y-y^{t}=0$, is added to the problem to ensure that the optimizer specified coupling variables match the actual computed value of the various coupling variables.

The optimization problems solved in this work are actually a hybrid of both MDF and IDF approaches. The analyses used herein can be decomposed into four disciplines: geometry, aerodynamics, structures and flight dynamics. The geometry discipline handles the wing surface manipulations and the computation of the CG, MAC and moment of inertia. The aerodynamics discipline handles the computation of the force and moment coefficients, as well as the stability derivatives for the aircraft. The structures discipline computes the bending coefficient and the flight dynamics discipline computes the static margin, CAP and damping ratio for the aircraft. The task of coupling the disciplines is simplified greatly by the fact that, for the analyses used in this work, there is no feedback between disciplines. Thus, the tightly coupled MDF architecture amounts to a sequenced evaluation of the disciplines. However, when using adjoint methods, coupling the derivatives of the various disciplines is not straightforward. This motivates the use of the IDF architecture, which requires only the individual discipline sensitivity analyses and greatly simplifies 


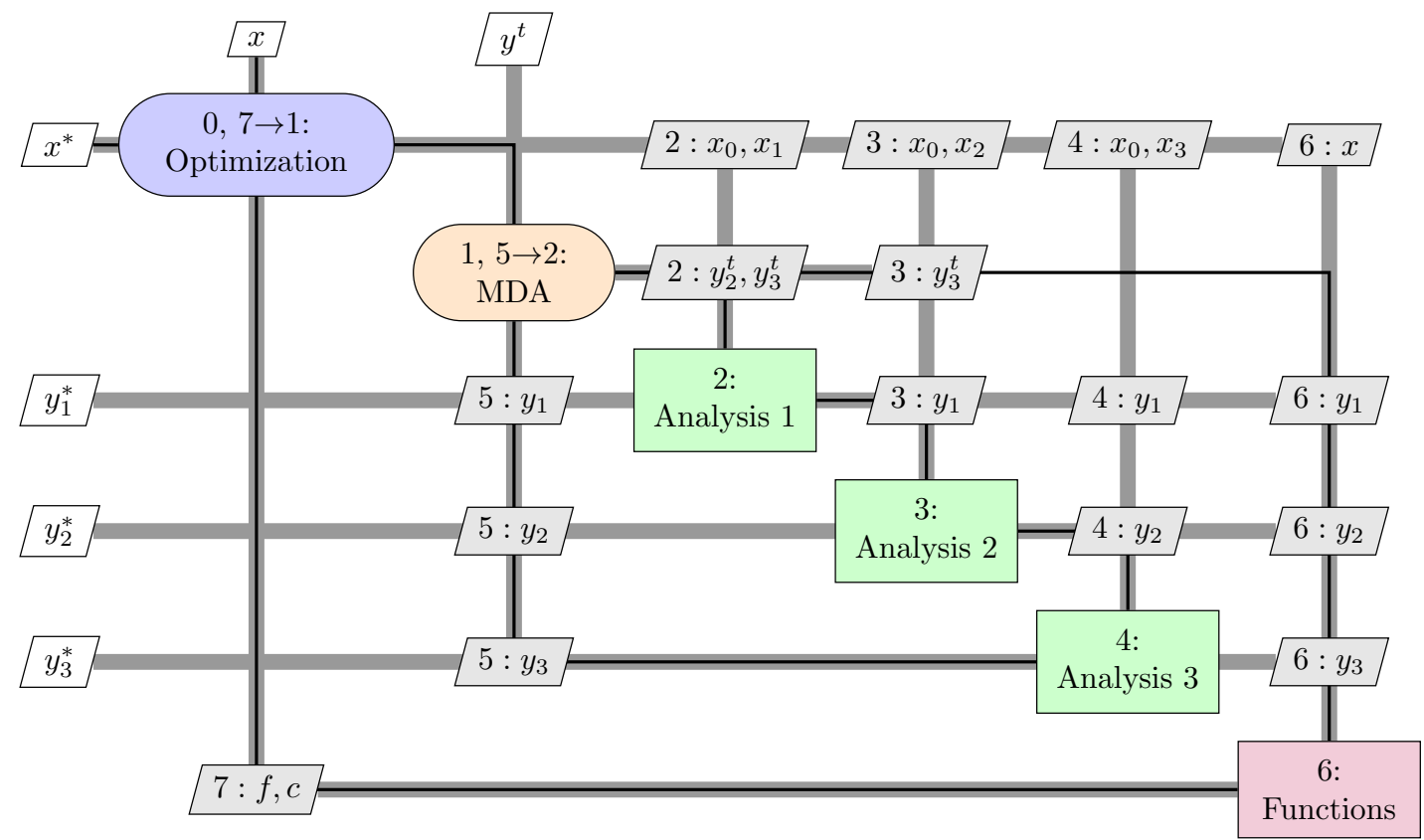

Figure 4. Generic MDF architecture [31]

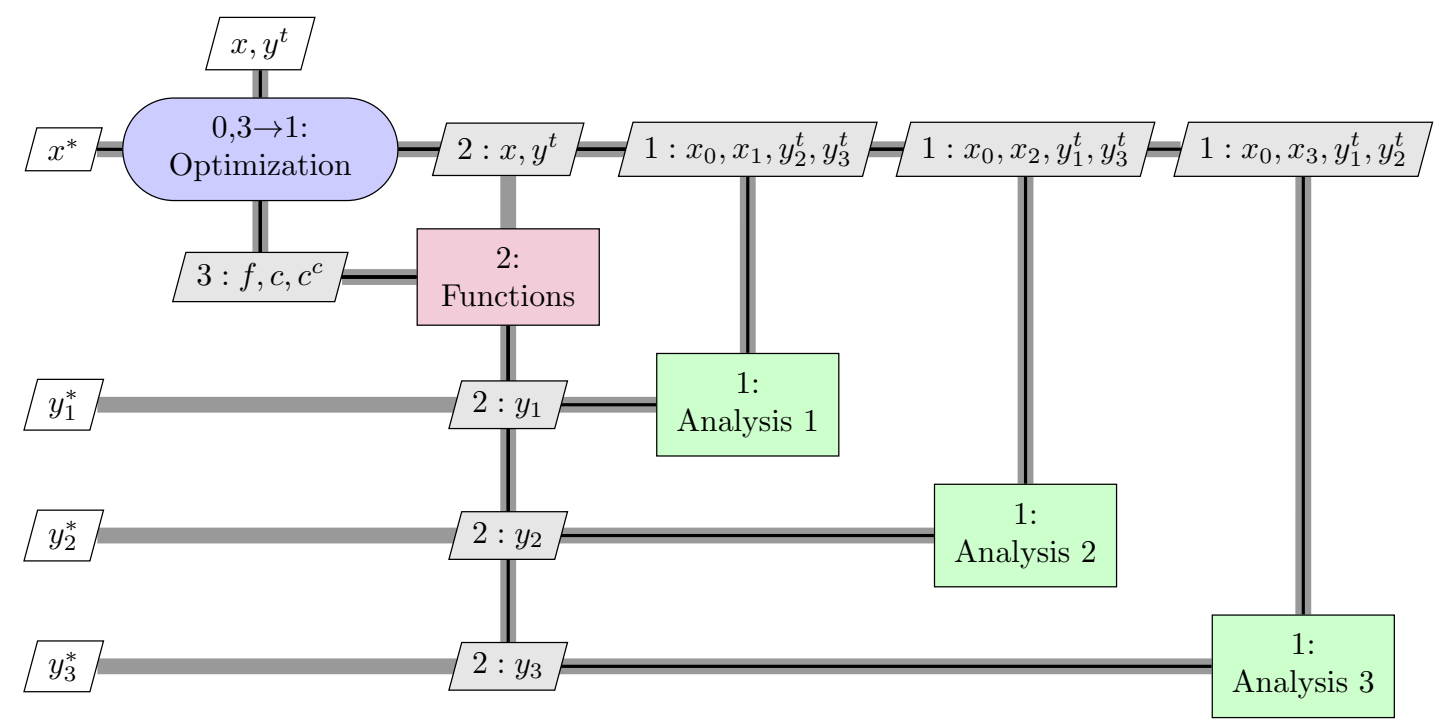

Figure 5. Generic IDF architecture [31] 
computation of accurate gradients.

Despite this apparent advantage, there are some disciplines for which tight coupling is a better choice. The coupling between the geometry and the aerodynamics, for example, requires a large number of coupling variables: the number of mesh points on the surface is typically in the order thousands or more. In an IDF architecture, this would add thousands of variables and constraints to the optimization problem, which would increase the complexity of the optimization problem unnecessarily. By tightly coupling the geometry surface to the aerodynamics, those coupling variables vanish from the optimization problem.

The other section where tight coupling makes sense is between the aerodynamics and structures. The bending coefficient calculation is based on six force and moment coefficients, of which only one , $C_{m_{z}}$, is already present in the optimization. Separating these two disciplines would require the solution of five additional adjoint problems per optimization iteration. By tightly coupling the aerodynamics and structures, this can be reduced to a single additional adjoint problem for the bending coefficient.

\section{Optimization Study Definition}

The baseline wing used for the current study is a straight, rectangular wing with a NACA0012 airfoil profile. The wing has a half span of three meters and a chord of one meter, giving the wing an aspect ratio of six. The wing has a taper ratio of one and a leading edge sweep angle of zero degrees. Details of the geometry are summarized in Table 2 This test case is based on the induced drag validation case proposed by Hicken and Zingg [7], who demonstrate that using this wing, with sections twisted about the trailing edge, one can reproduce the elliptical distribution outlined by lifting line theory. In particular, they highlight the use of a straight trailing edge, with spanwise sections twisted about the trailing edge, to minimize the impact of nonplanar effects in the wake. This configuration is used as the initial geometry in this set of optimizations so that the twist-only optimization outlined in Section B reproduces the elliptical result and can be used as a point of comparison for the remaining optimizations. This provides a means of quantifying the effect of the various stability constraints on the optimal solution. All of the optimization results presented are computed on an 1,105,920 cell mesh. The mesh has a C-O topology and is split into 32 blocks. The farfield boundary is approximately 15 chords from the wing and the off wall spacing of the mesh is $1 \times 10^{-3} \mathrm{~m}$ at the leading edge and $5 \times 10^{-4} \mathrm{~m}$ at the trailing edge.

\begin{tabular}{lr}
\hline \hline Parameter & Value \\
\hline Half wing area (m) & 3.0 \\
Half wing span (m) & 3.0 \\
Chord (m) & 1.0 \\
Leading edge sweep (deg.) & 0.0 \\
Taper ratio & 1.0 \\
Wing tip washout (deg.) & 0.0 \\
Wing dihedral & 0.0 \\
\hline
\end{tabular}

Table 2. Baseline wing: geometry specifications

\section{A. Design Variables}

Two sets of design variables are used: one involving only planform variables and one involving planform variables as well as 280 surface shape design variables. These surface shape design variables - the control points of the FFD volume - modify the surface shape of the wing and affect both the stream-wise and span-wise profile of the wing. Table 3 shows the primary design variables that alter shape and planform of the wing as well as the variables that affect the flow condition of the test case, while Table 4 shows the compatibility design variables introduced as a result of the hybrid IDF-MDF architecture used to solve the problems.

The primary design variables are:

Angle of attack, $\alpha$ : A variable to modify the free-stream flow direction and/or the mean grid velocity direction of the test case. This is the primary variable used to satisfy the $C_{L}$ constraint.

Section twist, $\theta_{i}$ : Variables to modify the twist values for individual sections along the wing. The sections rotate about the reference axis, which for this study is located at the wing trailing edge. These variables primarily affect the spanwise lift distribution, which in turn affects the induced drag created by the wing. 


\begin{tabular}{lcrr}
\hline \hline Design variable & Symbol & Lower bound & Upper bound \\
\hline Angle of attack (deg.) & $\alpha$ & -15 & 15 \\
Section twist (deg.) (9 sections) & $\theta_{i}$ & -10 & 10 \\
Area (m) & $A_{1}$ & 2.9 & 3.2 \\
Span (m) & $b$ & 2.0 & 3.2 \\
Sweep (deg.) & $\lambda$ & 0 & 42.5 \\
Center of gravity variable & $\mathrm{CG}_{\%}$ & -1 & 1 \\
$I_{z z}$ Modifier & $P_{I_{z z}}$ & 0.5 & $10,20,30$ \\
FFD control points: y-offset (m) & $\Delta_{\text {FFD }}$ & -0.05 & 0.05 \\
\hline
\end{tabular}

Table 3. Primary design variables and their bounds

\begin{tabular}{lcrr}
\hline \hline Design variable & Symbol & Lower bound & Upper bound \\
\hline Moment reference location (m) & $x_{\text {CG }}^{t}$ & -20 & 20 \\
Rotation point (m) & $x_{\mathrm{CG}}^{t}$ & -20 & 20 \\
Chord (m) & $c_{\text {root }}^{t}$ & 0.5 & 1.4 \\
Target MAC (m) & MAC & -5 & 5 \\
Target drag coefficient & $C_{D}^{t}$ & -2 & 2 \\
Target lift curve slope & $C_{L}^{t}$ & 0 & 20 \\
Target moment curve slope & $C_{m_{\alpha}}^{t}$ & -20 & 0 \\
Target $\dot{\alpha}$ derivative & $C_{m_{\dot{\alpha}}}^{t}$ & -20 & 20 \\
Target pitch derivative & $C_{m_{q}}^{t}$ & -20 & 0 \\
\hline
\end{tabular}

Table 4. Compatibility design variables and their bounds

Area, $A_{1}$ : The planform area of half the wing. This value is divided equally among the sections specified in PyACDT. Note that this value remains essentially constant. It varies to allow the calculated value of area, $A_{2}$, meet the constraint. This is necessary because $A_{2}$ varies slightly with wing twist and sweep.

Span, $b$ : The span of the half wing. This value is divided equally among the sections specified in PyACDT. Since the area is essentially fixed this variable also determines the chord of the wing. The span has a strong impact on both the induced drag and the root bending moment coefficient of the wing.

Sweep, $\lambda$ : The leading edge sweep of the wing. This variable affects both the wave drag and the root bending moment coefficient of the wing.

Center of gravity variable, $\mathbf{C G}_{\%}$ : A variable that controls the $x$ location of the aircraft center of gravity. This value represents the value of the location of the center of gravity in terms of a percentage of the distance between the front and rear spars at the MAC. Modifying this variable is equivalent to modifying $x_{\mathrm{CG}}$ and is used to provide a nicely scaled range of values for the optimizer to work with.

$I_{z z}$ modifier, $P_{I_{z z}}$ : A factor that multiplies the pitch moment of inertia. The base value of moment of inertia is calculated using the method described in Section D. This variable is a direct multiplier of that calculated value.

FFD control points: $y$-offset, $\Delta_{\text {FFD }}$ : Variables controlling the vertical motion of the FFD control points. These variables modify the shape of the wing surface and have a significant impact on the pitching moment of the wing as well as the wave drag caused by the wing.

In addition to the primary variables, we use the following compatibility variables:

Moment reference location, $x_{\mathbf{C G}}^{t}$ : An IDF target value that provides the $x$ location of the reference point used to calculate the moment coefficient, $C_{m}$, and all related quantities. This value is constrained to be equal to $x_{\mathrm{CG}}$ at the optimal solution.

Rotation point, $x_{\mathbf{C G}}^{t}$ : An IDF target value that provides the $x$ location of the rotation point for the flow solutions that involve a pitching motion. This value is constrained to be equal to the $x_{\mathrm{CG}}$ at the optimal solution.

Chord, $c_{\text {root }}^{t}$ : An IDF target variable for the root chord length. This variable is used to scale the allowable root bending moment coefficient, $C_{b_{r e f}}$, and is constrained to be equal to the value of root chord calculated in PYACDT. 
Target MAC, MAC ${ }^{t}$ : An IDF target variable for the reference chord length used in the CFD solver. Constrained to be equal to the MAC computed from the conceptual geometry definition at the optimal solution.

Target drag coefficient, $C_{D}^{t}$ : A variable resulting from the IDF architecture. The variable is used as an input to the various stability disciplines. The value is constrained to be equal to the drag coefficient predicted by the CFD solver at the optimal solution.

Target lift curve slope, $C_{L_{\alpha}}^{t}$ : A variable resulting from the IDF architecture. The variable is used as an input to the various stability disciplines. The value is constrained to be equal to the $C_{L_{\alpha}}$ value predicted by the CFD solver at the optimal solution.

Target moment curve slope, $C_{m_{\alpha}}^{t}$ : A variable resulting from the IDF architecture. The variable is used as an input to the various stability disciplines. The value is constrained to be equal to the $C_{m_{\alpha}}$ value predicted by the CFD solver at the optimal solution.

Target $\dot{\alpha}$ derivative, $C_{m_{\dot{\alpha}}}^{t}$ : A variable resulting from the IDF architecture. The variable is used as an input to the various stability disciplines. The value is constrained to be equal to the $C_{m_{\dot{\alpha}}}$ value predicted by the CFD solver at the optimal solution.

Target pitch derivative, $C_{m_{q}}^{t}$ : A variable resulting from the IDF architecture. The variable is used as an input to the various stability disciplines. The value is constrained to be equal to the $C_{m_{q}}$ value predicted by the CFD solver at the optimal solution.

Note that not all design variables are used for all cases. A number of the variables listed are present as a function of the IDF architecture mentioned previously and are, therefore, only present when the associated disciplines are included in the problem statement.

\section{B. Reference Problems \\ 1. Baseline Problem}

As a first step in the study, a problem is formulated to find the angle of attack, $\alpha$, and CG location, $x_{\mathrm{CG}}$, that yield a trimmed aircraft at the target value of $C_{L}$ for each Mach number. These problems are formulated as:

$$
\begin{aligned}
\text { minimize } & C_{D} \\
\text { w.r.t. } & \alpha, x_{\mathrm{CG}} \\
\text { subject to } & C_{L_{\mathrm{ref}}}-C_{L} \leq 0 \\
& C_{m}=0
\end{aligned}
$$

This is illustrated in Figure 6. Note that the lift coefficient constraint is formulated as an inequality constraint rather than an equality constraint. Because induced drag is proportional to $C_{L}^{2}$, reducing $C_{L}$ also reduced drag. Therefore, the optimizer will drive $C_{L}$ as close to zero as possible, making an upper limit on $C_{L}$, i.e. an equality constraint, unnecessary.

\section{Twist Optimization}

As a second benchmark, a twist-only optimization is performed for the subsonic - Mach $=0.5-$ case. This problem is designed to reproduce the elliptical result from lifting line theory and is formulated as:

$$
\begin{aligned}
\text { minimize } & C_{D} \\
\text { w.r.t. } & \alpha, \theta_{i}, \mathrm{CG}_{\%}, x_{\mathrm{CG}}^{t} \\
\text { subject to } & C_{L_{\mathrm{ref}}}-C_{L} \leq 0 \\
& C_{m}=0 \\
& x_{\mathrm{CG}}^{t}-x_{\mathrm{CG}}=0
\end{aligned}
$$

The data dependencies and solution process are shown in Figure 7. In this optimization, twist variables $\theta_{i}$ are added to control the lift distribution, and the full CG calculation is added to control the location of the CG. Note that the aircraft is trimmed during the optimization by forcing the pitch moment coefficient about the CG, $C_{m}$, to be zero. However, since the location of the $\mathrm{CG}$ is allowed to move - through the variable $\mathrm{CG}_{\%}$ - the aircraft can be trimmed without changing the aerodynamic shape. 


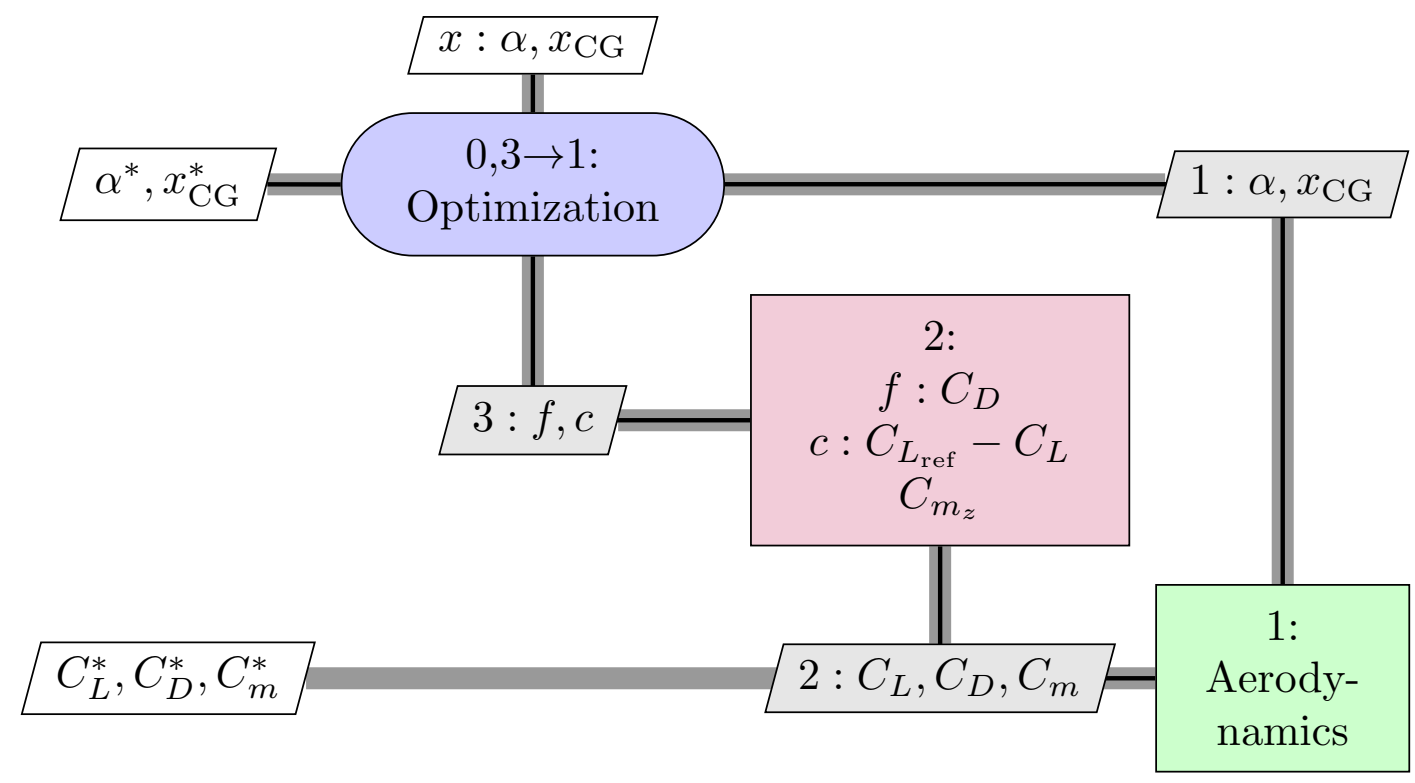

Figure 6. XDSM for the baseline problem

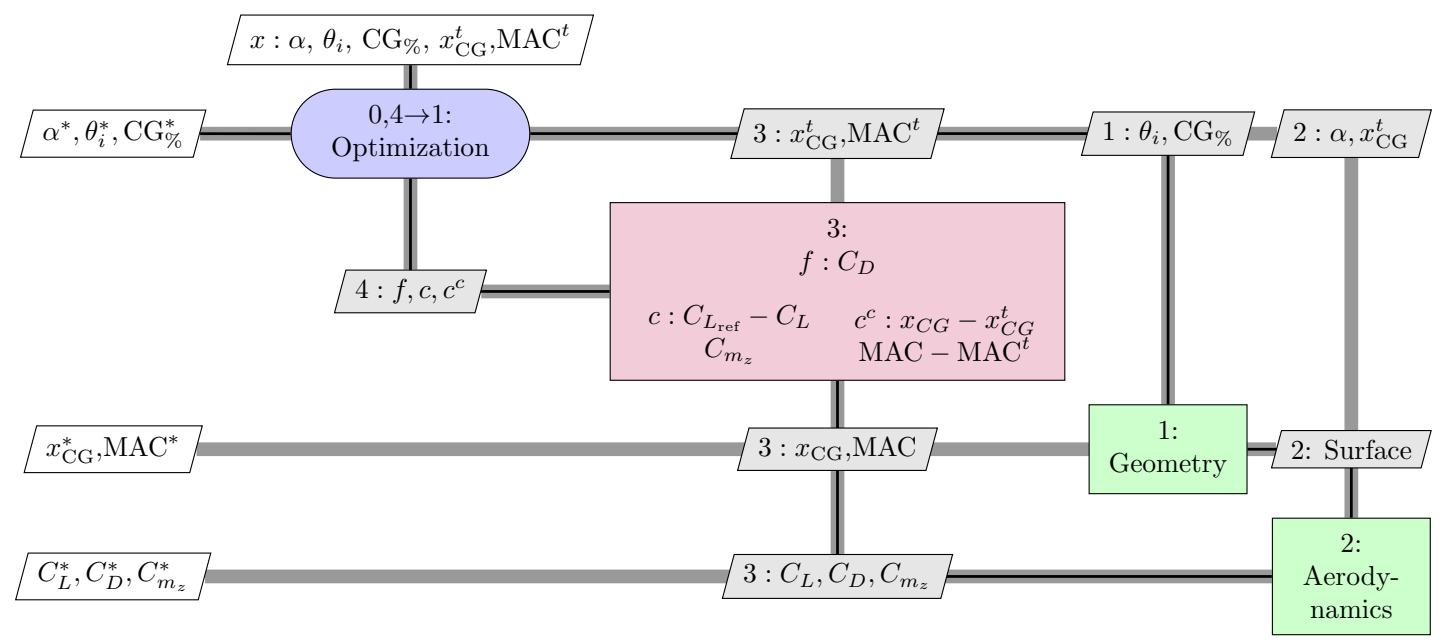

Figure 7. XDSM for the twist optimization problem 


\section{Bending Moment Constrained Optimizations}

As a third and final benchmark, an aerodynamic optimization problem with a structural constraint is considered. In this problem, span $(b)$, sweep $(\lambda)$ and area $\left(A_{1}\right)$ are added as additional design variables and a root bending moment constraint, $C_{b}$, is added to keep these variables within sensible bounds. The value of the bending coefficient at the root is that of the elliptical optimal solution at Mach $=0.5$. Additional compatibility constraints for the root chord, $c_{\text {root}}$, and the calculated area, $A_{2}$, have also been added to account for the new flexibility added to the problem. This problem is formulated as:

$$
\begin{aligned}
\operatorname{minimize} & C_{D} \\
\text { w.r.t. } & \alpha, \theta_{i}, A_{1}, b, \lambda, \mathrm{CG}_{\%}, \Delta_{\mathrm{FFD}}, c_{\mathrm{root}}^{t}, x_{\mathrm{CG}}^{t}, \mathrm{MAC}^{t} \\
\text { subject to } & C_{L_{\mathrm{ref}}}-C_{L} \leq 0 \\
& C_{m}=0 \\
& C_{\mathrm{b}}-C_{\mathrm{b}_{\text {ref }}}=0 \\
& A_{2}-A_{\text {ref }}=0 \\
& x_{\mathrm{CG}}-x_{\mathrm{CG}}^{t}=0 \\
& \mathrm{MAC}-\mathrm{MAC}^{t}=0 \\
& c_{\text {root }}-c_{\mathrm{root}}^{t}=0
\end{aligned}
$$

Figure 8 depicts the data and process flow for this formulation in graphical form.

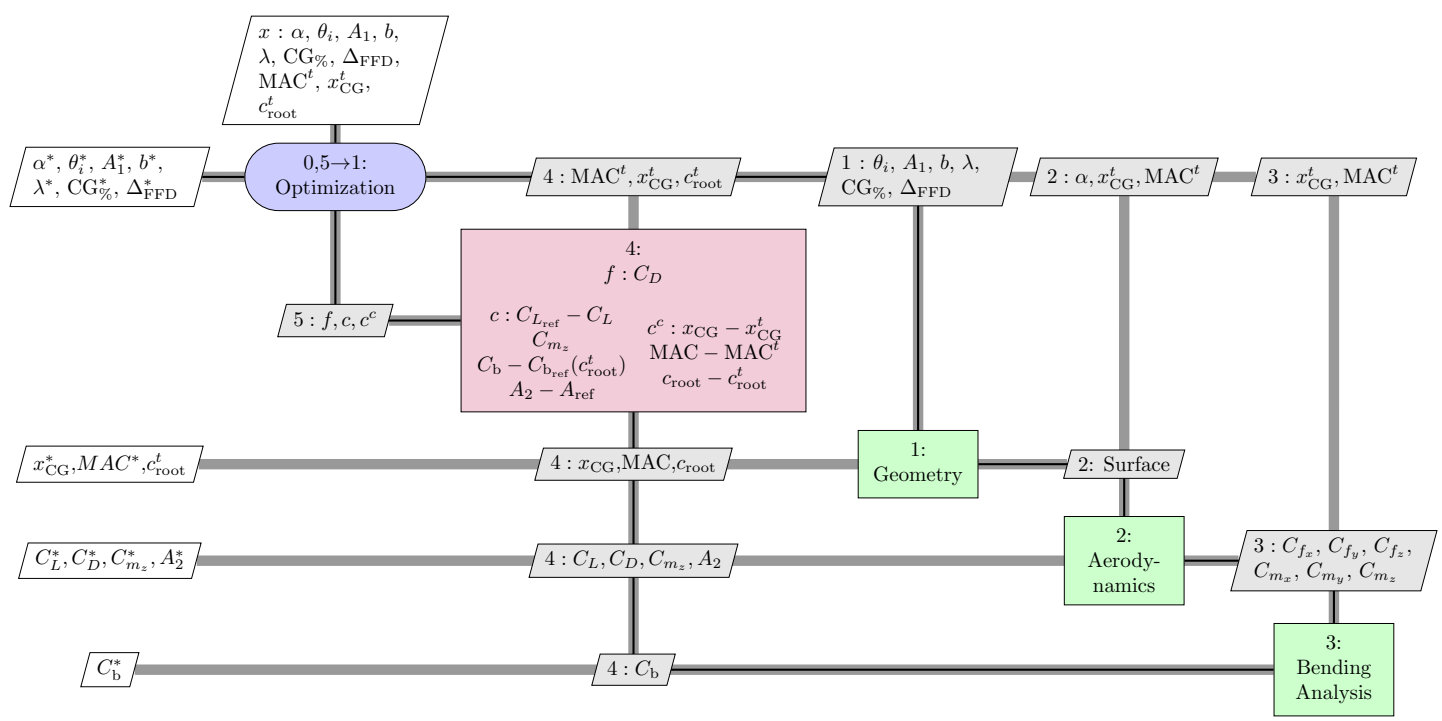

Figure 8. XDSM for the root bending moment constrained problem 


\section{Static Stability Constrained Problems}

The first stability constrianed formulation is based on static margin. By introducing this constraint we can ensure that the optimal design has the desired static margin. The static margin formulation is as follows:

$$
\begin{aligned}
\text { minimize } & C_{D} \\
\text { w.r.t. } & \alpha, \theta_{i}, A_{1}, b, \lambda, \mathrm{CG}_{\%}, \Delta_{\mathrm{FFD}} \\
& c_{\text {root }}^{t}, x_{\mathrm{CG}}^{t}, \mathrm{MAC}^{t}, C_{L_{\alpha}}^{t}, C_{m_{\alpha}}^{t} \\
\text { subject to } & C_{L_{\text {ref }}}-C_{L} \leq 0 \\
& C_{m}=0 \\
& K_{n_{\text {ref }}}-K_{n} \leq 0 \\
& C_{\mathrm{b}}-C_{\mathrm{b}_{\text {ref }}}=0 \\
& A_{2}-A_{\text {ref }}=0 \\
& x_{\mathrm{CG}}-x_{\mathrm{CG}}^{t}=0 \\
& \mathrm{MAC}-\mathrm{MAC}=0 \\
& c_{\text {root }}-c_{\text {root }}^{t}=0 \\
& C_{L_{\alpha}}-C_{L_{\alpha}}^{t}=0 \\
& C_{m_{\alpha}}-C_{m_{\alpha}}^{t}=0
\end{aligned}
$$

Note that in the above formulation, $K_{n}$ is the static margin and that additional constraints have been added for $C_{L_{\alpha}}^{t}$ and $C_{m_{\alpha}}^{t}$ corresponding to the additional IDF target variables that have been added for the computation of the static margin constraint. A graphical depiction of solution process for this formulation is shown in Figure 9.

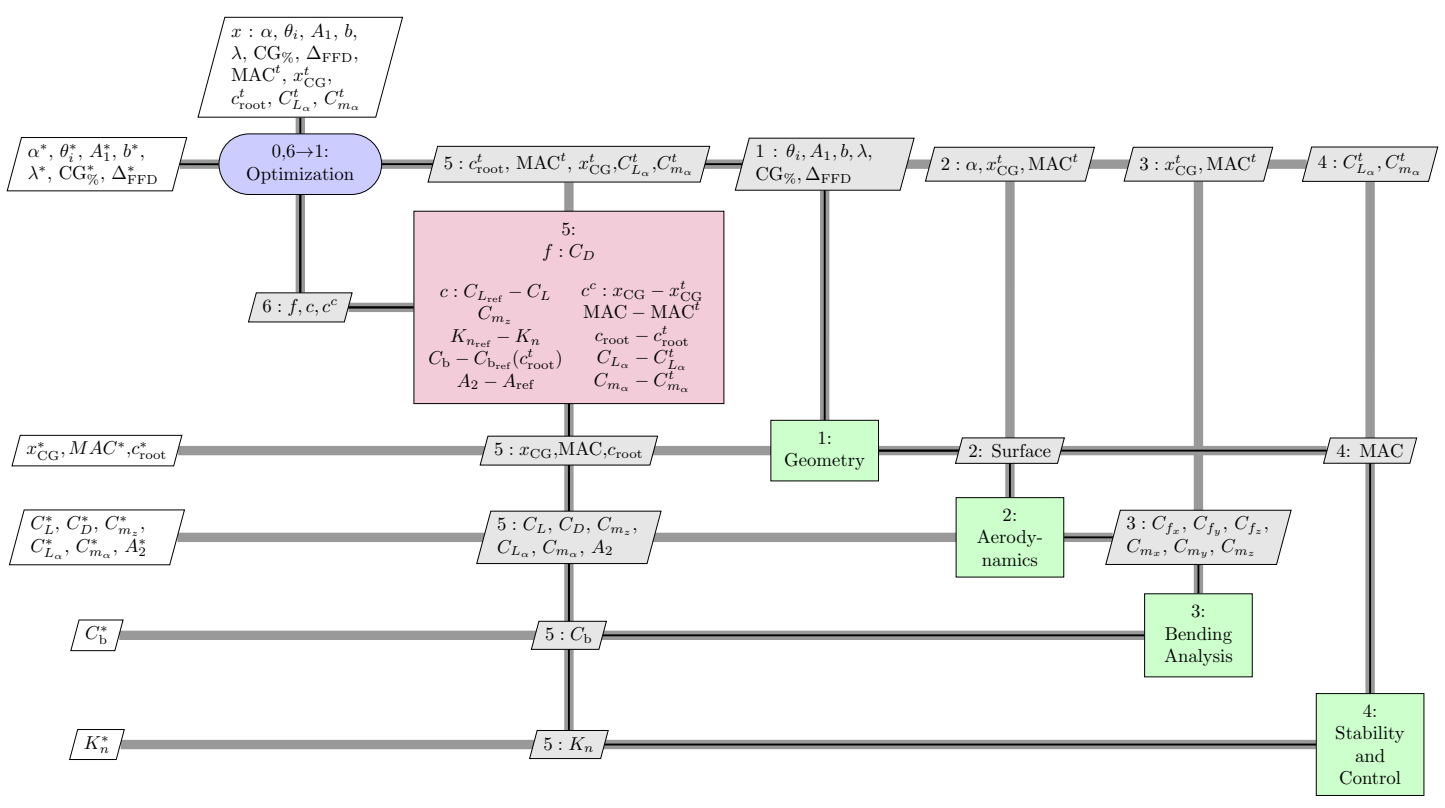

Figure 9. XDSM for the $K_{n}$ constrained problem 


\section{Dynamic Stability Constrained Problems}

The dynamic stability formulation used in this work is based on the CAP parameter described in Section II. This formulation builds on the previously described static margin formulation and can be written as:

$$
\begin{aligned}
\text { minimize } & C_{D} \\
\text { w.r.t. } \quad x= & \alpha, \theta_{i}, A_{1}, b, \lambda, \mathrm{CG}_{\%}, \Delta_{\mathrm{FFD}}, c_{\mathrm{root}}^{t}, x_{\mathrm{CG}}^{t}, \mathrm{MAC}^{t}, \\
& C_{D}^{t}, C_{L_{\alpha}}^{t}, C_{m_{\alpha}}^{t}, C_{m_{\dot{\alpha}}}^{t}, C_{m_{q}}^{t} \\
& C_{L_{\mathrm{ref}}}-C_{L} \leq 0 \\
& C_{m}=0 \\
& K_{n_{\mathrm{ref}}}-K_{n} \leq 0 \\
& 0.085 \leq C A P \leq 3.6 \\
& 0.3 \leq \zeta_{s p} \leq 2.0 \\
& A_{2}-A_{\mathrm{ref}}=0 \\
& x_{\mathrm{CG}}-x_{\mathrm{CG}}^{t}=0 \\
& \mathrm{MAC}-\mathrm{MAC}=0 \\
& c_{\mathrm{root}}-c_{\mathrm{root}}^{t}=0 \\
& C_{D}-C_{D}^{t}=0 \\
& C_{L_{\alpha}}-C_{L_{\alpha}}^{t}=0 \\
& C_{m_{\alpha}}-C_{m_{\alpha}}^{t}=0 \\
& C_{m_{\dot{\alpha}}}-C_{m_{\dot{\alpha}}}^{t}=0 \\
& C_{m_{q}}-C_{m_{q}}^{t}=0
\end{aligned}
$$

Relative to the static margin optimization, two primary constraints have been added, a CAP constraint and a $\zeta_{s p}$ constraint. These constraints define the appropriate limits for the dynamic stability parameters. The range of allowable $\mathrm{CAP}$ values constrains the allowable short-period frequency, and the damping ratio, $\zeta_{s p}$, is constrained directly. Also, IDF constraints have been added for $C_{D}^{t}, C_{m_{\dot{\alpha}}}^{t}$, and $C_{m_{q}}^{t}$ to reflect the addition of these variables for the computation of the dynamic stability parameters. A graphical depiction of the solution process for this formulation is shown in Figure 10.

\section{Results}

The following section summarized the results and findings of the optimization study. We present the results of the various optimiziation formulations in order, identifying the effect of each constraint on the optimal solution. The various optimizations are conducted at $\mathrm{M}=0.5,0.7$ and 0.85 respectively to compare the effects the subsonic and transonic flow regimes on the solution.

\section{A. Reference Problems}

1. Baseline cases

The solutions for Mach = 0.5, 0.7 and 0.85 are shown in Figures 11 through 13. These solutions show that the solutions at the three different Mach numbers each have different characteristics. The solution at $\mathrm{M}=0.5$ is fully subsonic, with a correspondingly peaky $C_{p}$ distribution on the airfoil. Both the neutral point of the wing and the required center of gravity to trim are just slightly forward of the wing quarter chord. The $\mathrm{M}=0.7$ case is just starting into the transonic regime and the inner half of the wing is exhibiting a weak shockwave near the leading edge. The location of the center of gravity to trim the aircraft is slightly further forward than in the $\mathrm{M}=0.5$ case, but is still near the quarter chord. The neutral point is also shifted slightly forward. The $\mathrm{M}=0.85$ case is fully in the transonic regime and exhibits a strong shockwave on the aft half of the airfoil. As a result, the neutral point and the center of gravity location to trim are significantly farther back, near the half chord. Note that in each case the the free stream parameters (pressure and density) have been scaled such that the dynamic pressure $q$ is the same for all cases. 


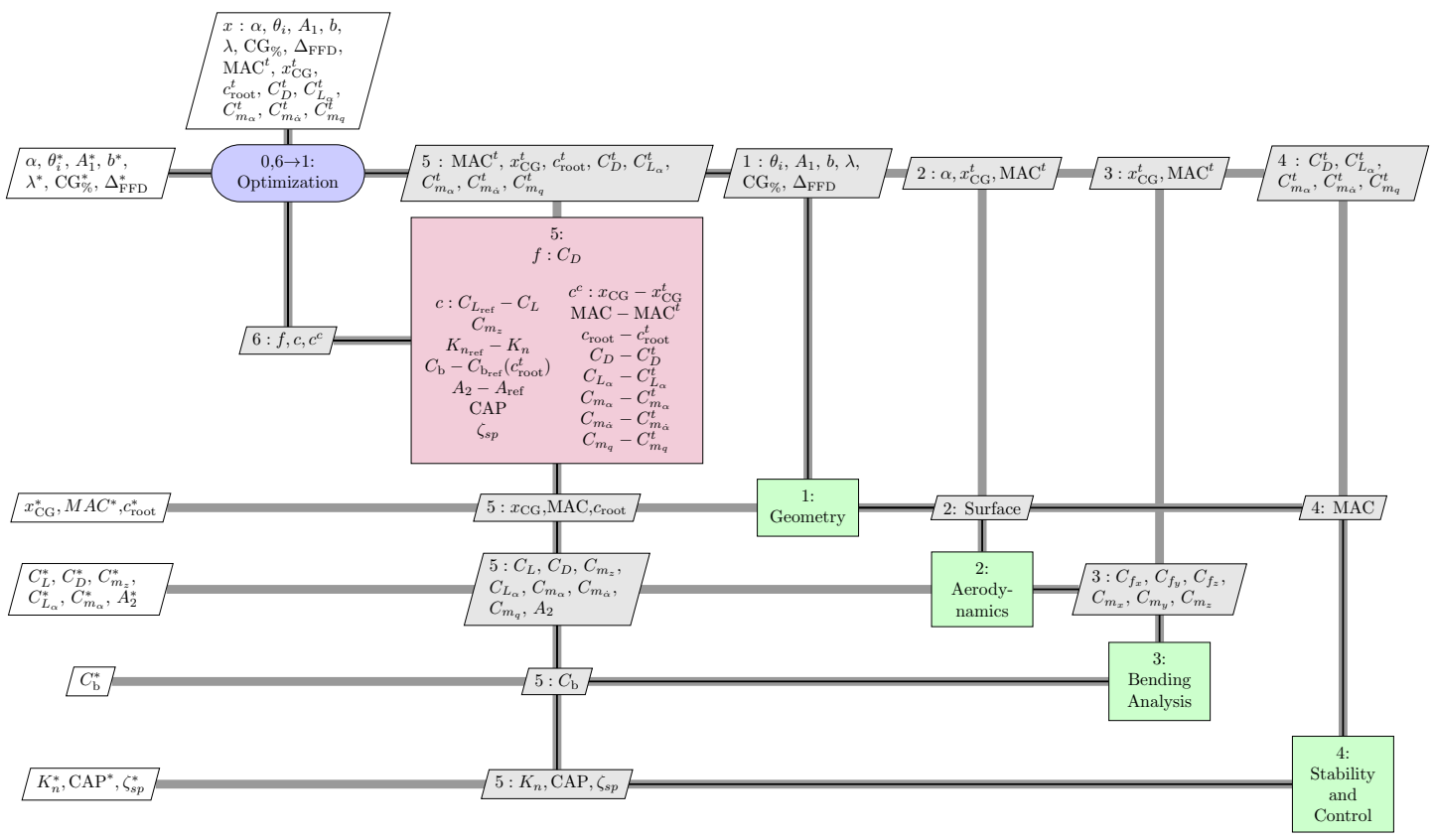

Figure 10. XDSM for the CAP constrained problem

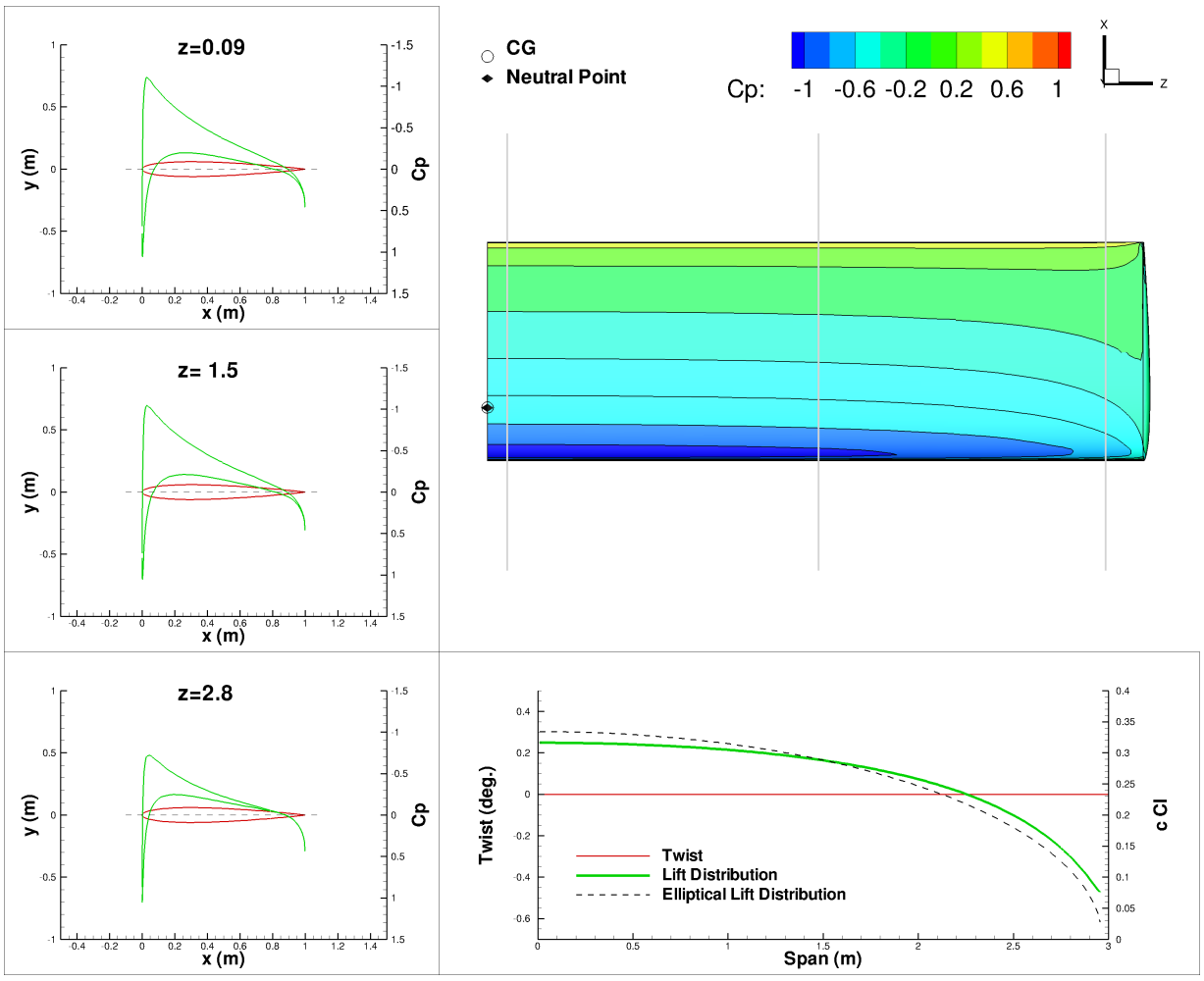

Figure 11. Baseline problem: $\mathbf{M = 0 . 5}, e=0.964$ 


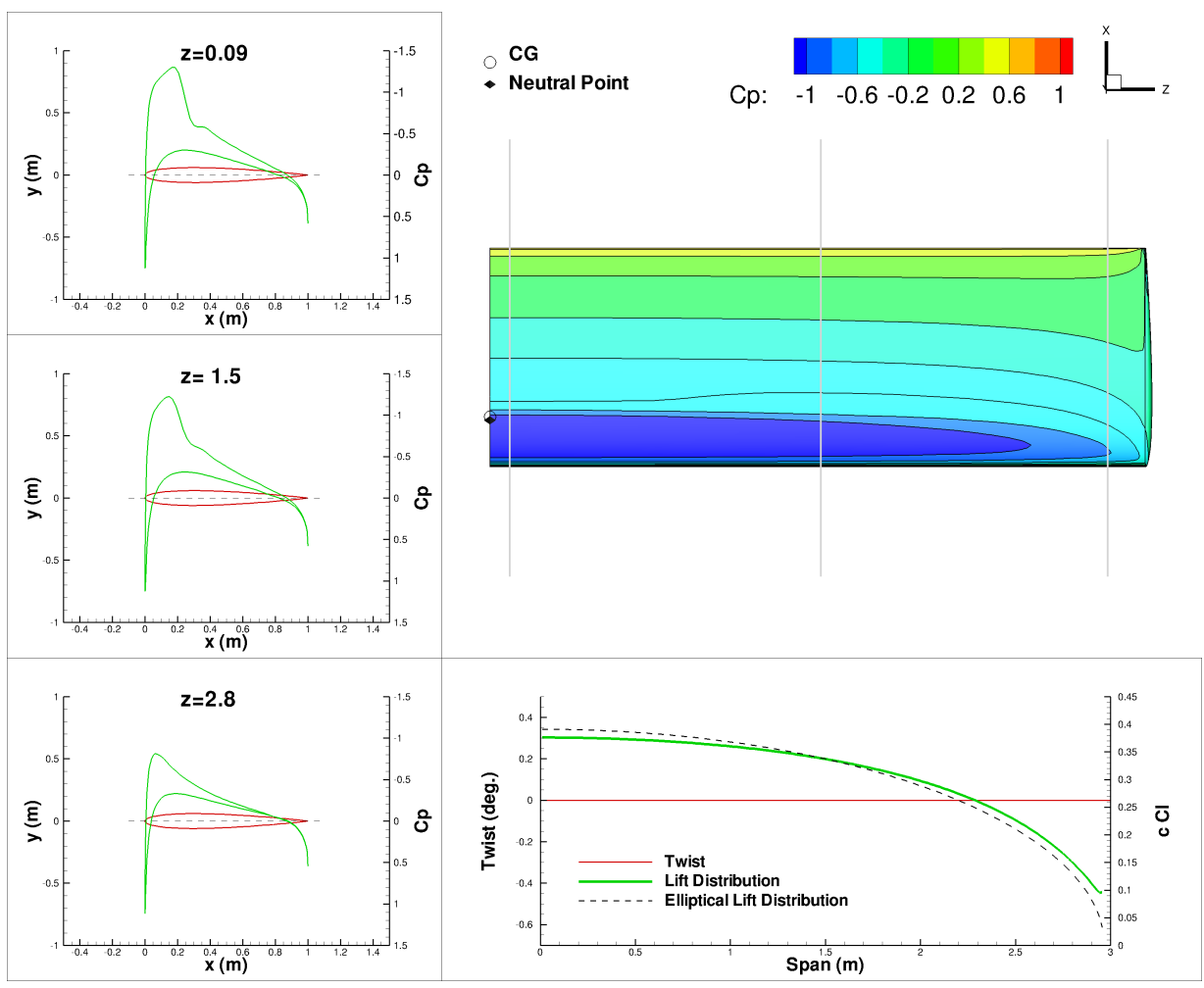

Figure 12. Baseline problem: $M=0.7$

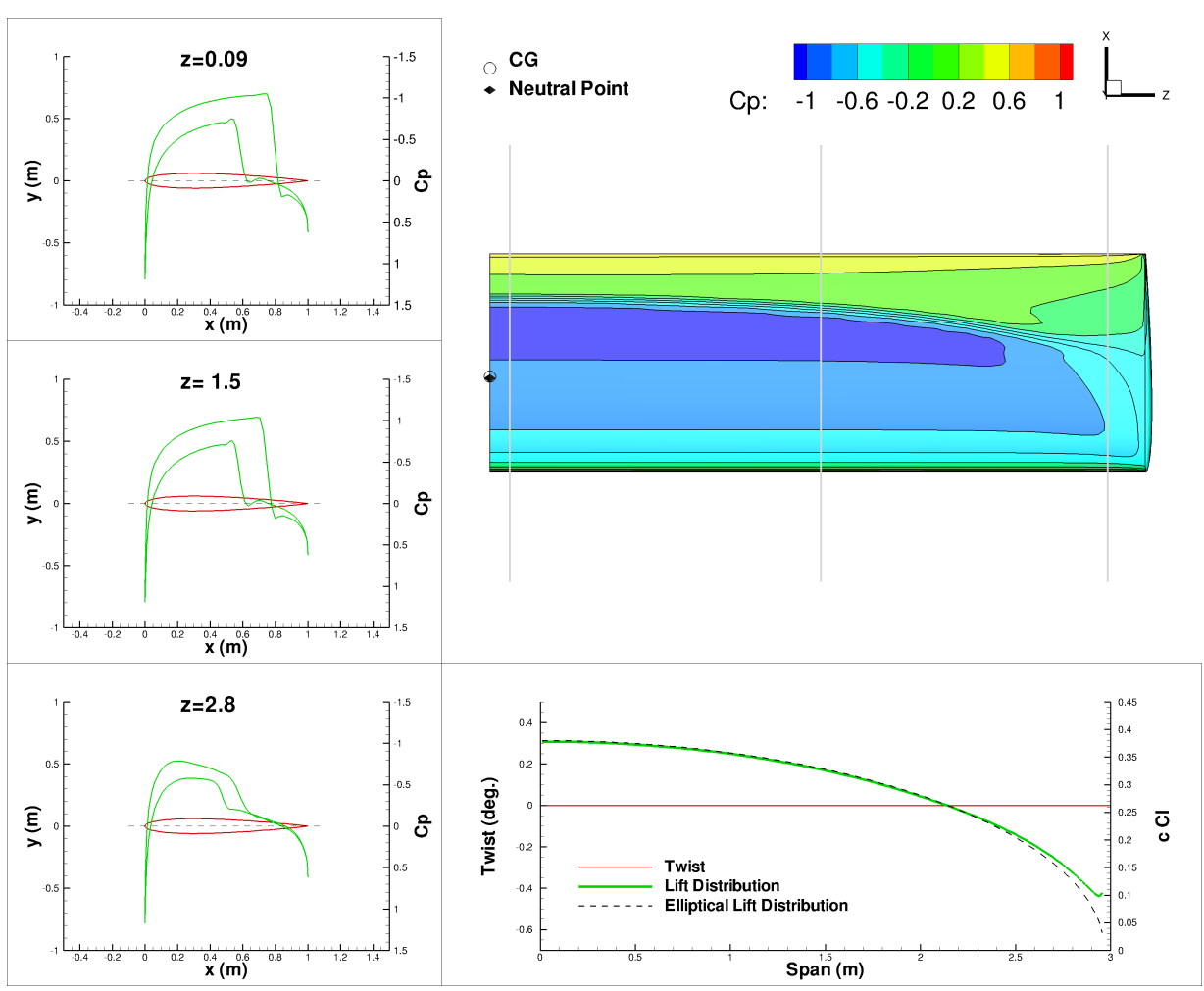

Figure 13. Baseline problem: $M=0.85$

19 of 34 


\section{Twist-Only Case}

The twist-only formulation yields the solution shown in Figure 14. The lift distribution achieved with this solution

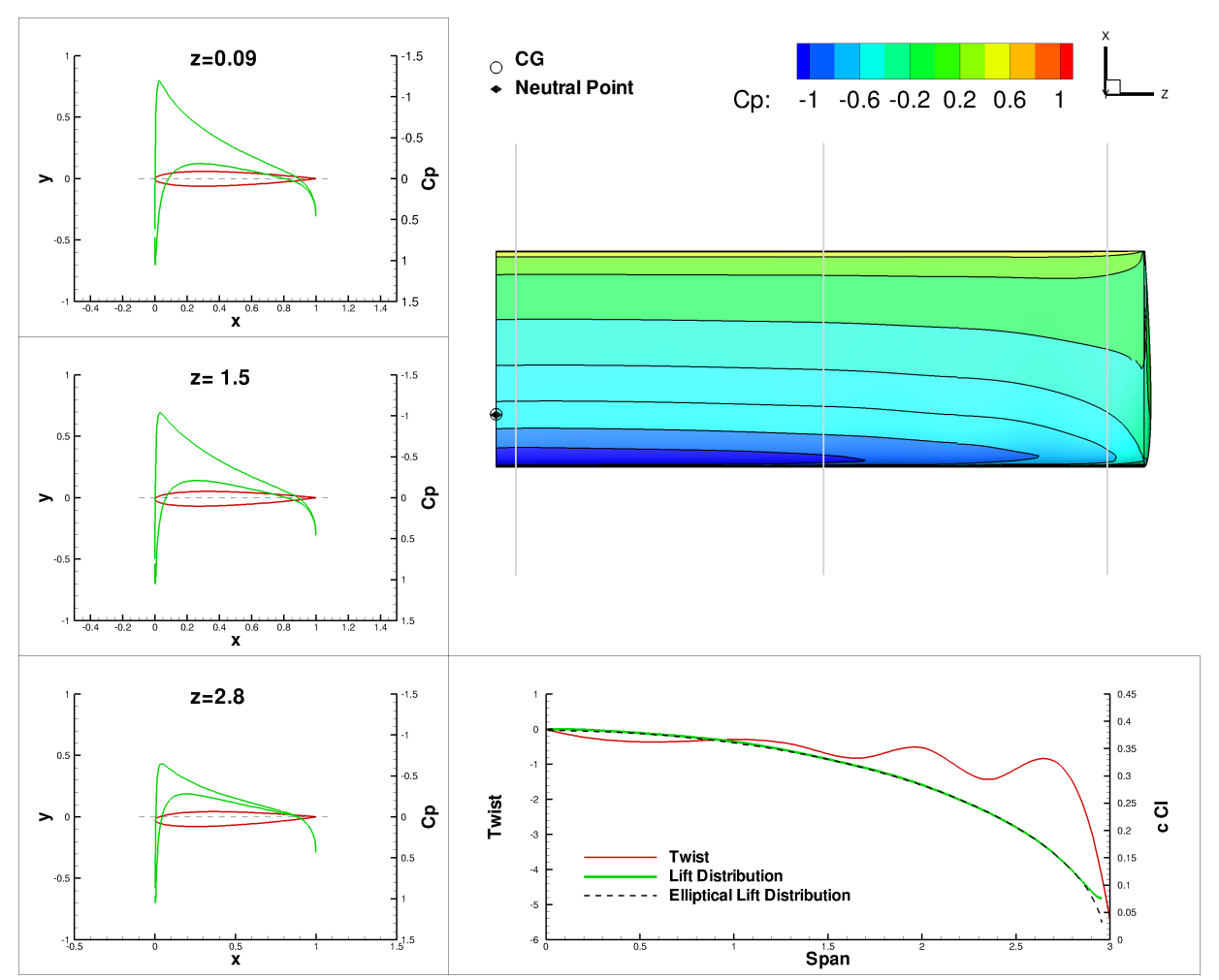

Figure 14. Twist optimization: $\mathbf{M = 0 . 5}, e=0.977$

clearly shows an optimal solution that closely approximates the elliptical solution. The exception to this is the area near the tip. This location consists of complex three-dimensional flows that violate the assumptions used to derive the elliptical result; therefore, it is expected that the lift distribution in this region does not match the elliptical distribution.

\section{Bending Moment Constrained Cases}

The root bending moment constrained optimizations have the same design variable flexibility as the stability constrained cases described in Sections B and C. Therefore, they are able to produce shapes with lower drag than those cases with stability constraints. These optimizations provide the best indication of how much performance is sacrificed to ensure that the stability constraints are satisfied. The results for the planform-only optimizations are shown in Figures 15 and 16, while the results including shape variables are shown in Figures 17 and 18. Note that there is no shape optimization result for the $\mathrm{M}=0.5$ case, as the airfoil shape has little impact on the drag of a subsonic wing in inviscid flow.

Comparing the elliptical optimum solution shown in Figure 14 to the bending constrained case at $\mathrm{M}=0.5$, shown in Figure 15, we observe some subtle, yet important differences. In the bending constrained case, the optimizer takes advantage of the span variable to reduce the induced drag. There is a visible extension in the span of the wing as compared to the original wing, which is shown as a black outline. This increase in span is limited by the allowable root bending moment and comes with a corresponding movement away from an elliptical lift distribution.

Looking at the $\mathrm{M}=0.7$ case, shown in Figure 16, the optimizer adds a significant amount of sweep to the wing. This causes a trade-off between induced drag and wave drag. The added sweep reduces the effective Mach number normal to the wing leading edge, thereby reducing the strength of the leading edge shock. With sufficient sweep, the Mach number normal to the leading edge of the wing is reduced below the critical Mach number, eliminating the shock wave and the corresponding wave drag. However, adding sweep increases the effective root bending moment. Thus, as sweep is added, the span must be reduced to meet the bending constraint. This is evident in the difference in span between the Mach $=0.5$ case shown in Figure 15 and the Mach $=0.7$ case shown in Figure 16.

When shape variables are added, the need for sweep to reduce wave drag is eliminated and, as shown in Figure 17, 


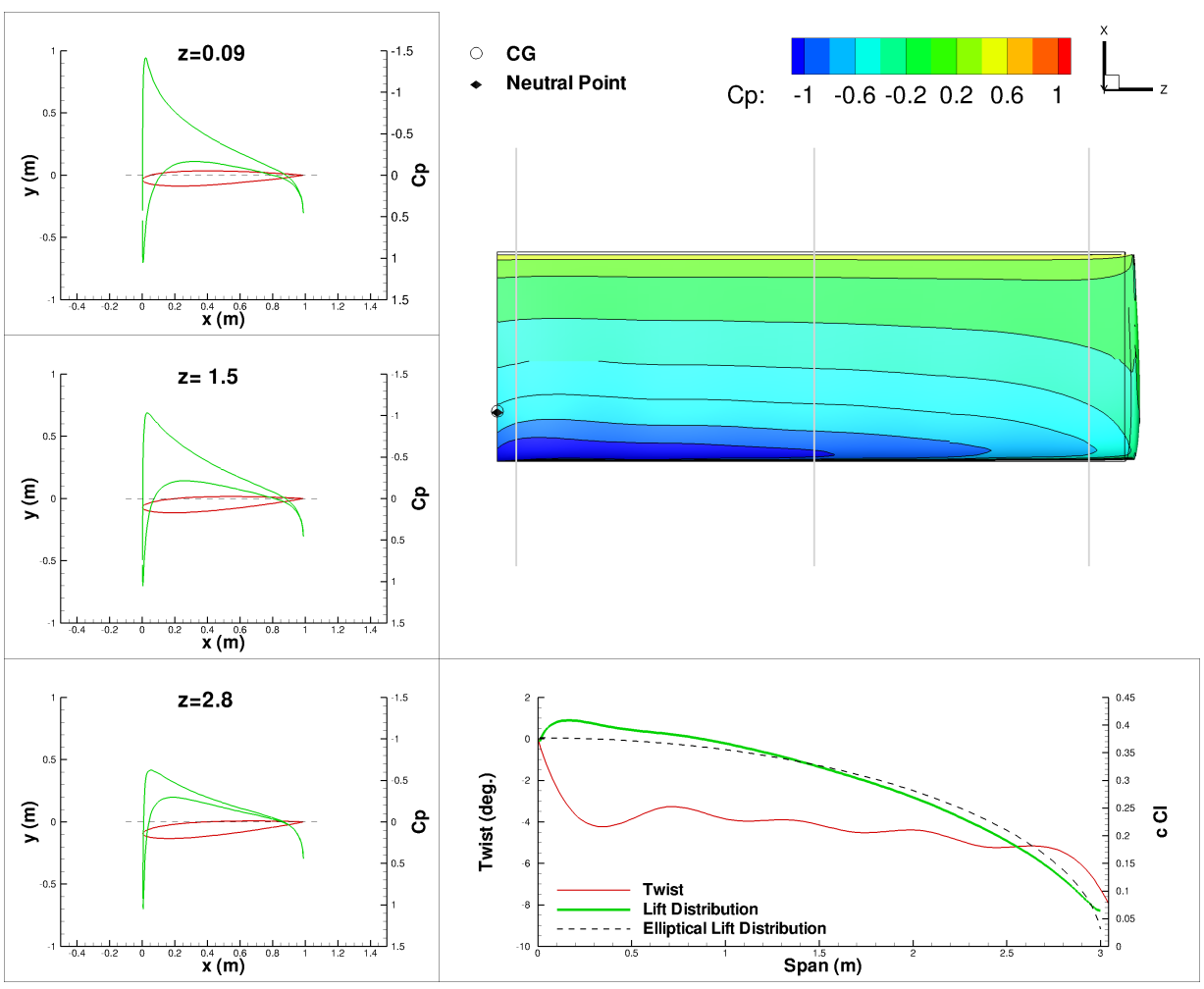

Figure 15. Bending moment constrained optimization: planform variables only: $\mathbf{M}=\mathbf{0 . 5}, e=0.964$

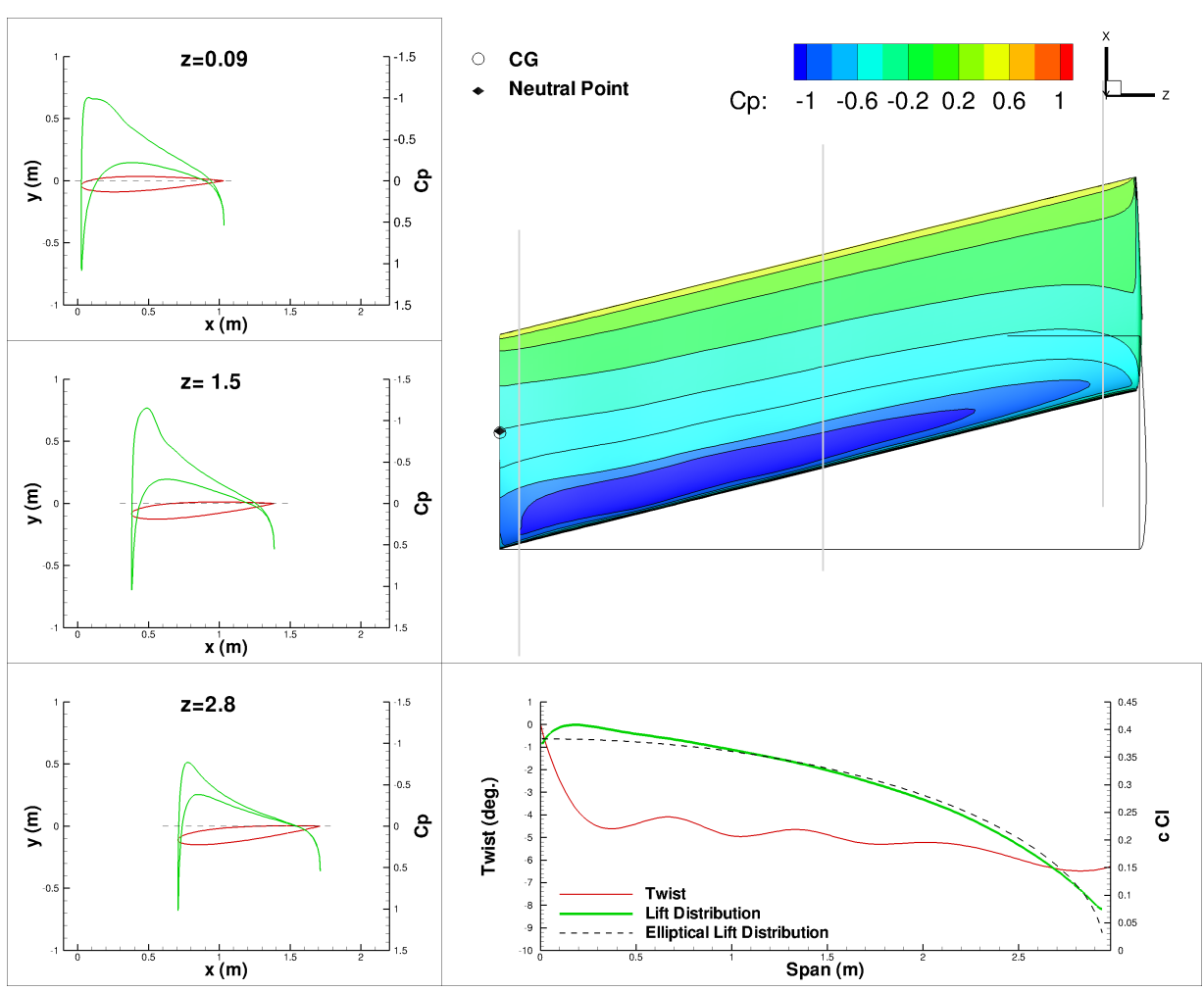

Figure 16. Bending moment constrained optimization: planform variables only: $M=0.7$ 


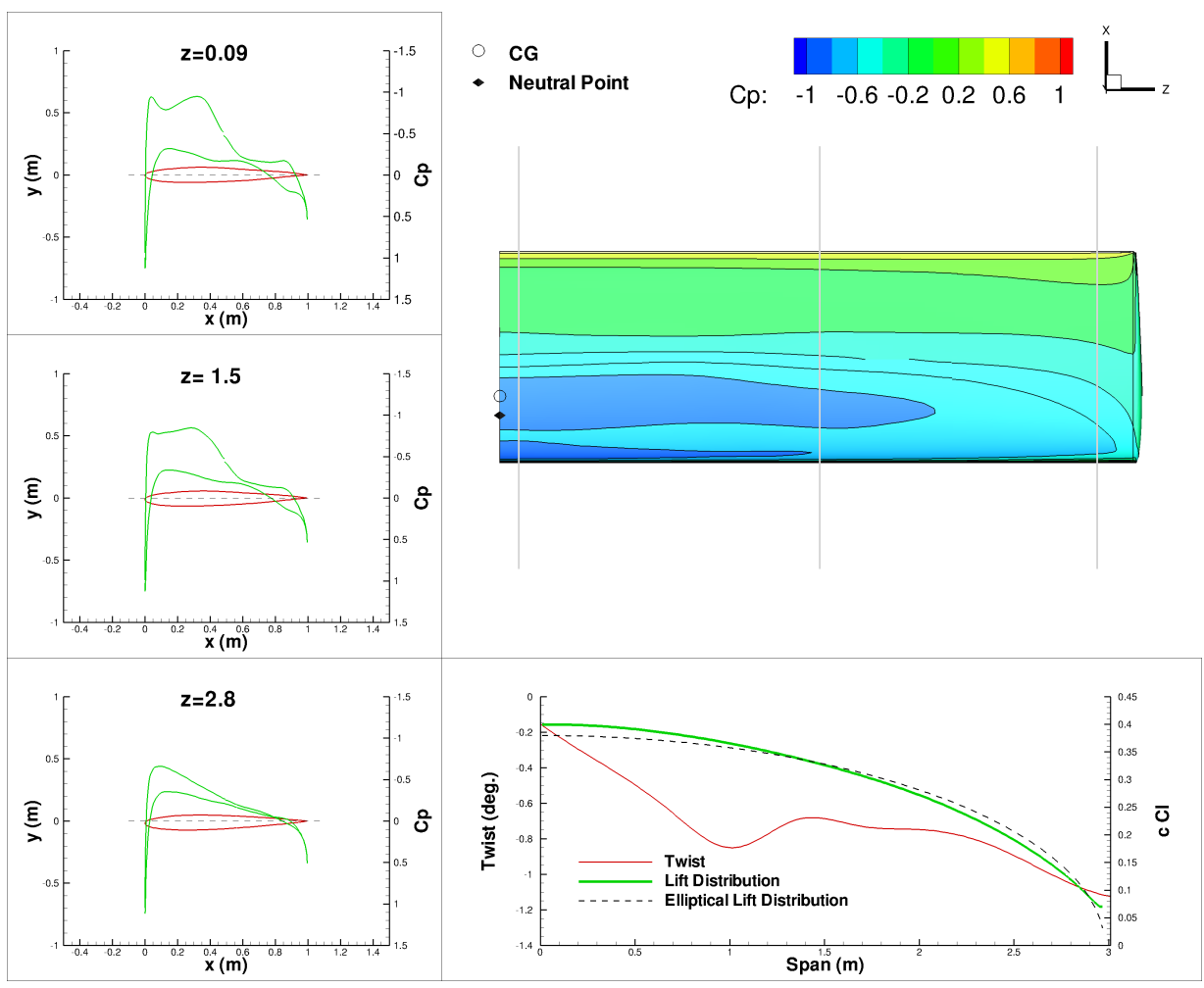

Figure 17. Bending moment constrained optimization: planform and shape variables: $M=0.7$

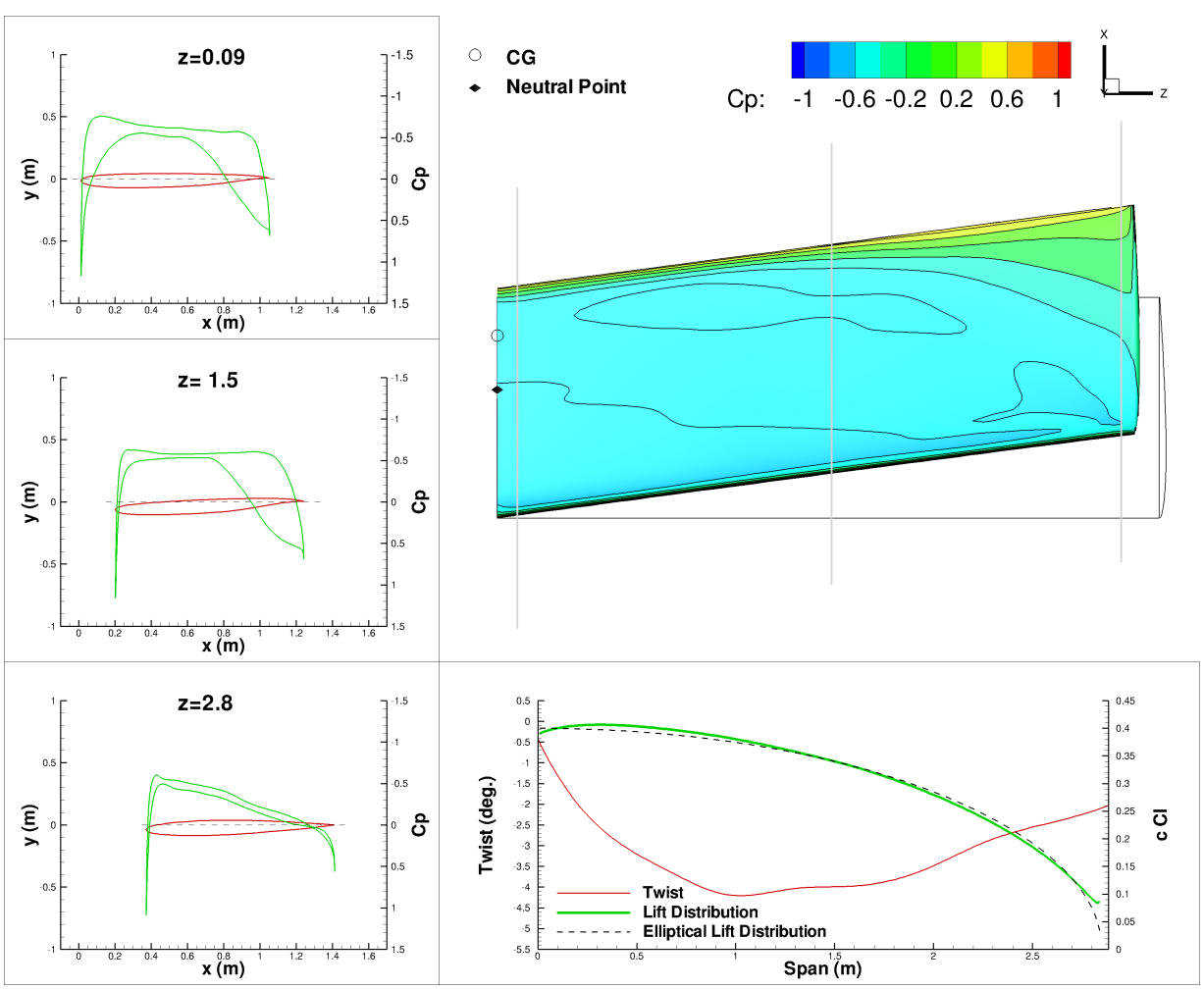

Figure 18. Bending moment constrained optimization: planform and shape variables: $M=0.85$ 
the optimizer does not sweep the wing, allowing for a larger span. Further, there is now a significant change in the $C_{p}$ distribution on the wing. The individual sections now show a rooftop pressure profile at the leading edge, allowing the maximum section $C_{p}$ to stay below the critical $C_{p}$ for the wing, thereby eliminating the wave drag on the wing. Note that the planform is now similar to the optimum $\mathrm{M}=0.5$ planform in Figure 15.

At $\mathrm{M}=0.85$, the optimizer produces the features typical of supercritical transonic airfoils for the individual airfoil sections. The sections show a rooftop $C_{p}$ profile and are highly aft loaded. However, even with these transonic airfoils, the wave drag is not entirely eliminated, so the optimizer introduces some sweep in the design to mitigate this effect. This added sweep causes a corresponding reduction in span to maintain the required bending moment. Note that because of the aft loaded nature of these airfoils, the neutral point of the wing is significantly forward of the required CG location for trimmed flight, leading to a statically unstable configuration.

\section{B. Static Stability Constrained Problems}

The planform-only, static margin constrained optimization at Mach $=0.5$, shown in Figure 19, shows an increase in sweep relative to the baseline case. The optimizer is adding sweep and wash-out to trim the aircraft for a more forward CG position. In this case, the magnitude of the changes is sufficient to generate the necessary separation between the neutral point and CG for a 5\% static margin. Once shape variables are added, this additional sweep is not necessary

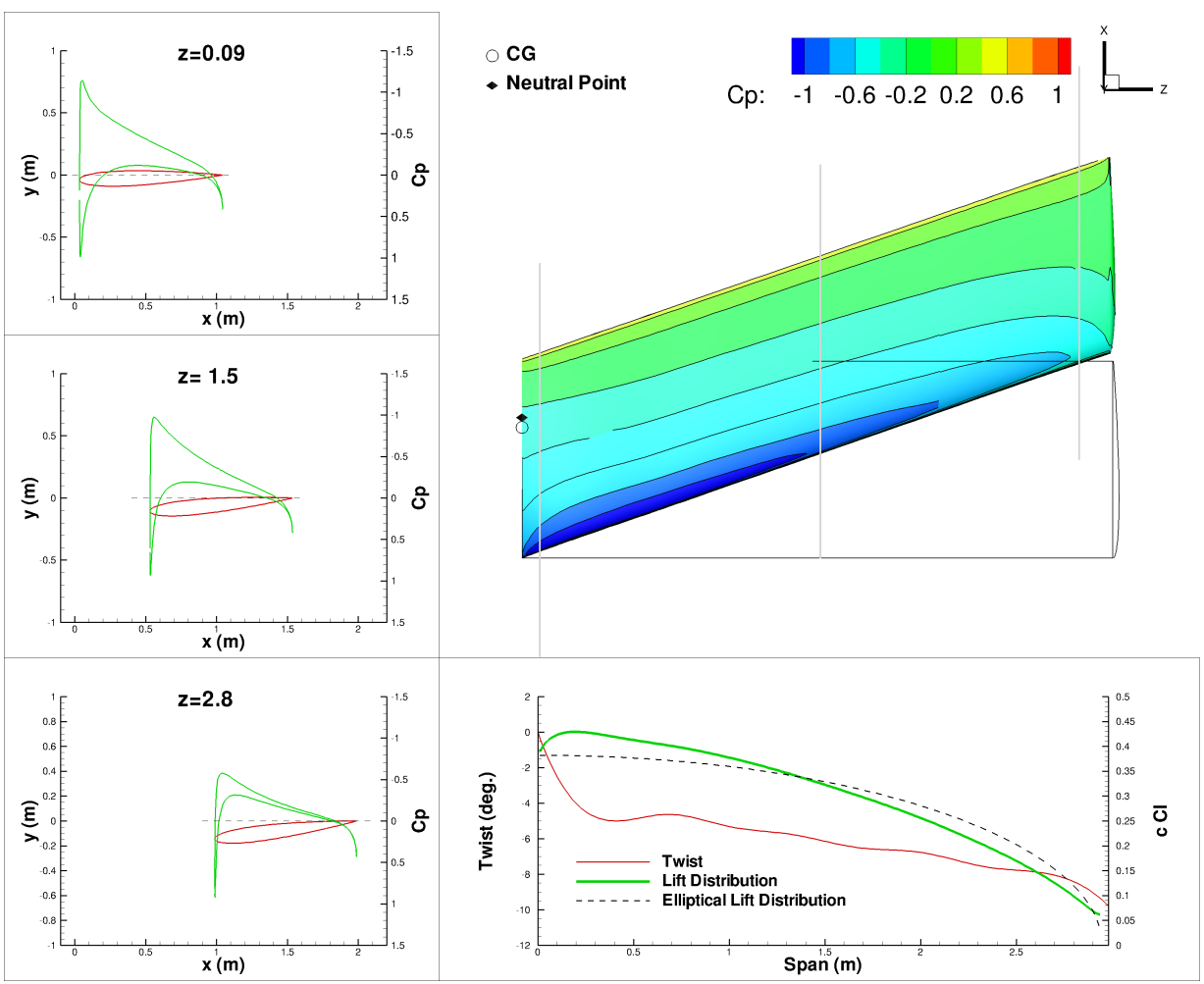

Figure 19. $K_{n}$ constrained optimization: planform variables only: $\mathbf{M = 0 . 5}, e=0.944$

to stabilize the aircraft. As shown in Figure 20, the optimizer is able to use the shape variables to modify the $C_{p}$ to achieve the same effect. In this case, the $C_{p}$ distribution, especially near mid-span, has developed negative lift near the trailing edge, which alters the $C_{m_{\mathrm{NP}}}$ of the wing. This allows the optimizer to shift the CG forward - stabilizing the wing - using $\mathrm{CG}_{\%}$ while still maintaining a trimmed state. Because the wing is in a stable trimmed state without sweep, there is no increase in the bending moment associated with sweep. Therefore the optimizer is again able to increase the span from the baseline value of three meters, reducing the resulting induced drag.

Looking at the planform-only optimization at Mach $=0.7$, the optimizer again adds sweep and twist to create separation between the neutral point of the wing and the CG location of the aircraft while maintaining a trimmed state. As can be seen in Figure 21 there is now visible separation between the neutral point and the CG location. Again, the addition of shape variables allows the optimizer to un-sweep the wing without sacrificing performance. As shown in Figure 22, the optimizer uses the shape variables to alter the $C_{p}$ distribution of the wing to trim the wing in a stable state. In this case, in addition to adding the negative lift near the trailing edge, the optimizer has flattened the $C_{p}$ 


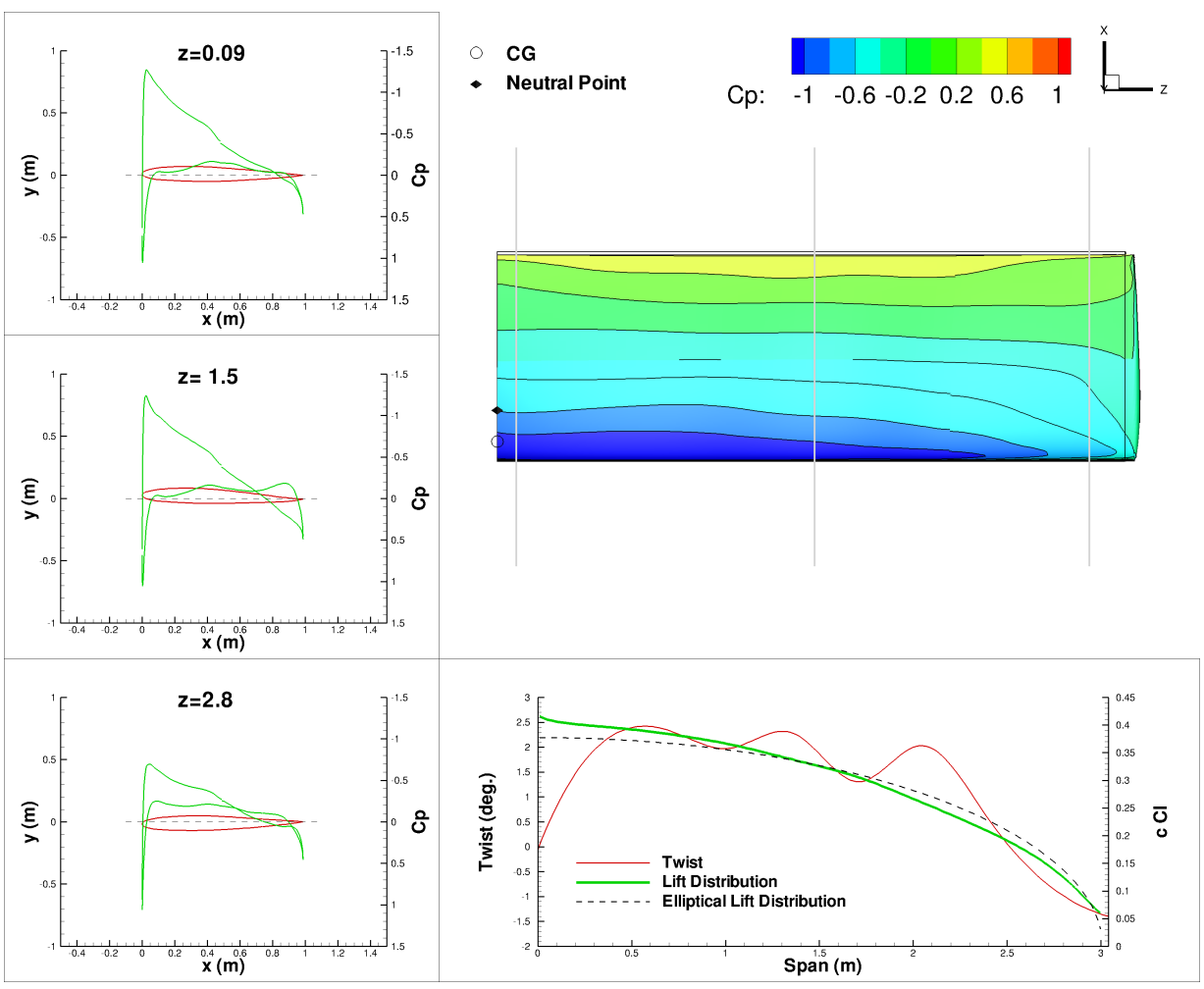

Figure 20. $K_{n}$ constrained optimization: planform and shape variables: $\mathbf{M}=\mathbf{0 . 5}, e=0.968$

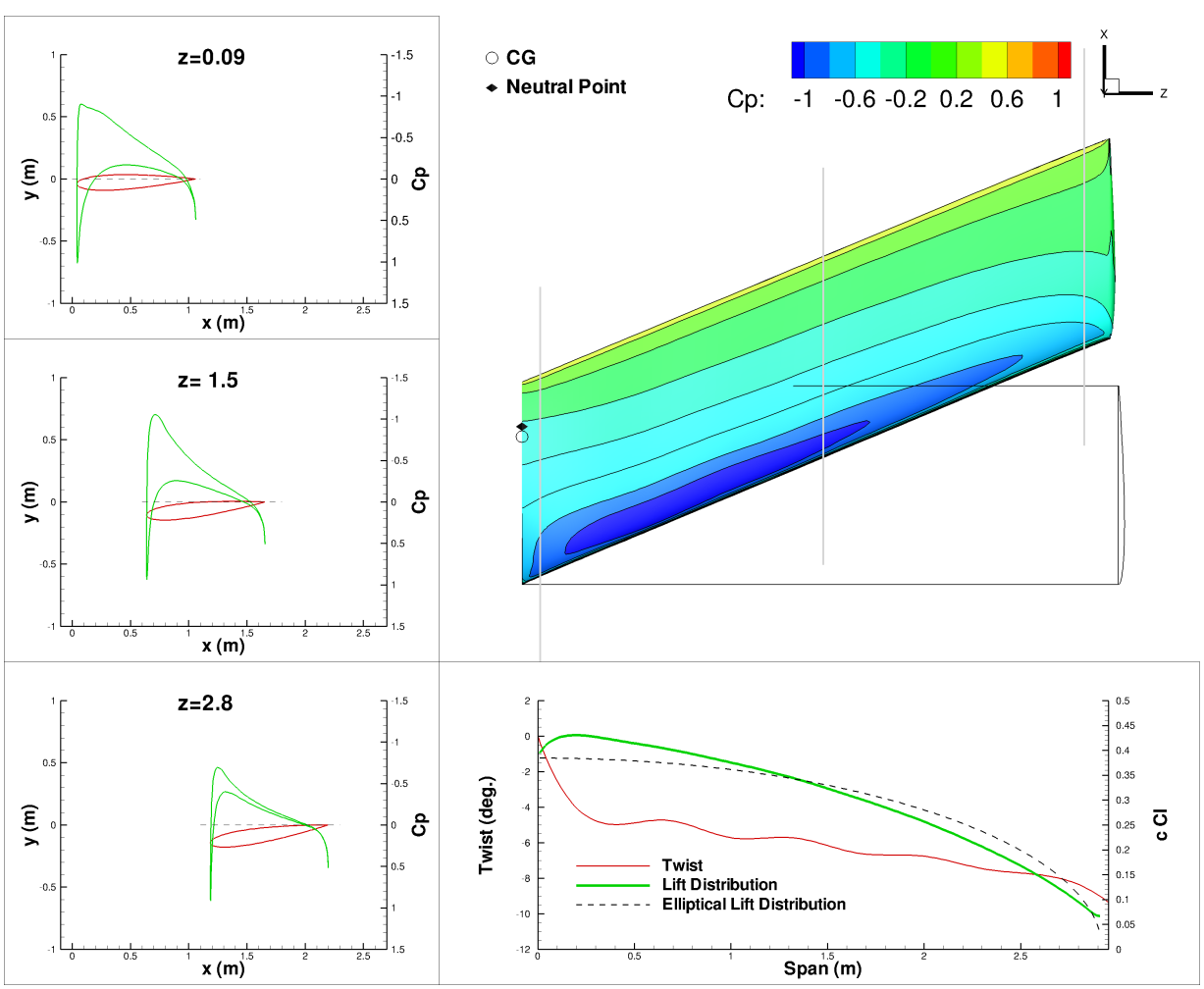

Figure 21. $K_{n}$ constrained optimization: planform variables only: $\mathbf{M = 0 . 7}$ 
distribution at the leading edge of the wing, eliminating the pressure peak and thereby reducing the wave drag. In this case the optimal solution has a very small amount of sweep.

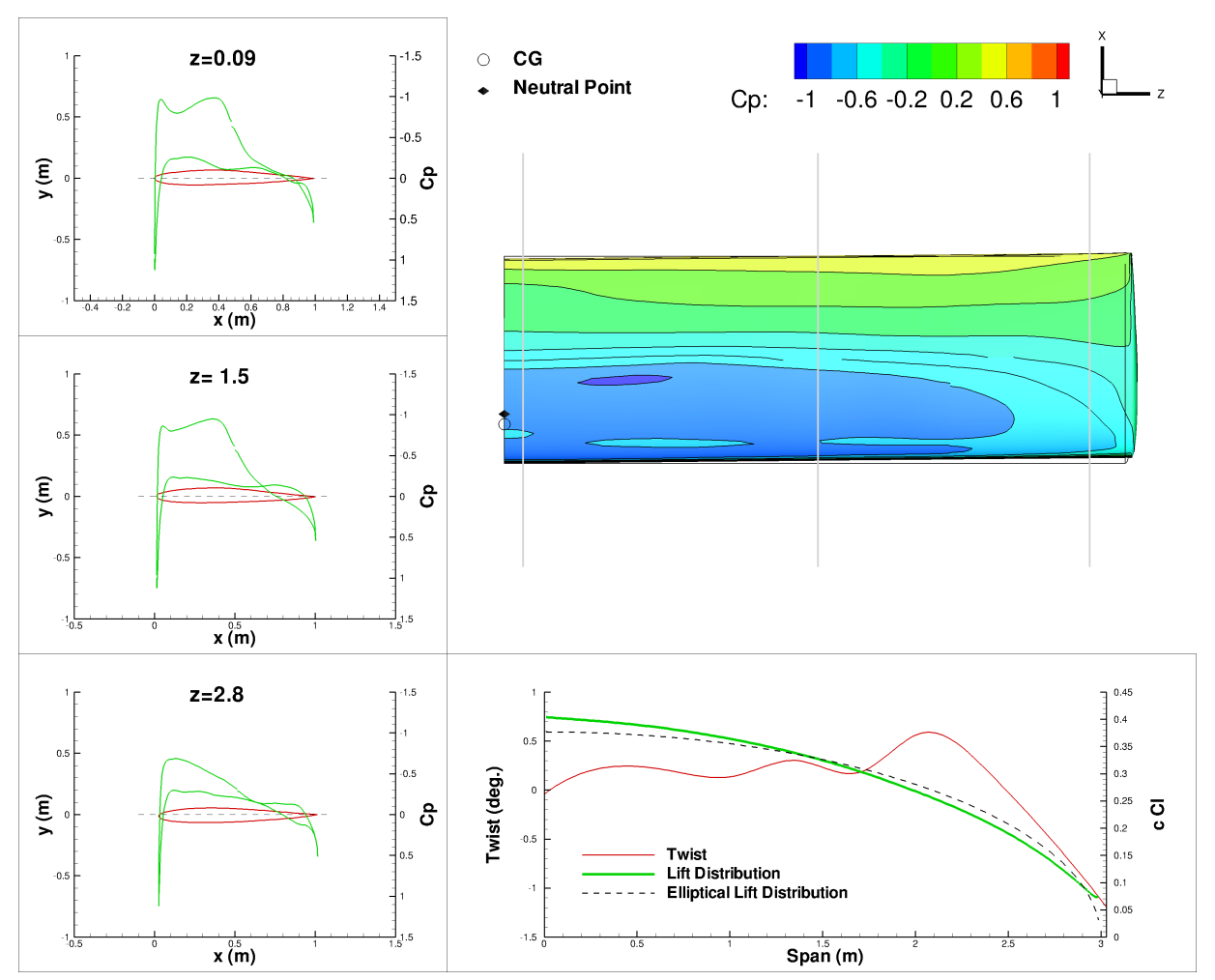

Figure 22. $K_{n}$ constrained optimization: planform and shape variables: $\mathrm{M=0.7}$

The Mach $=0.85$ case, shown in Figure 23, is physically a more challenging problem for the optimizer to solve. As in the previous bending constrained case, the optimizer attempts to reduce the wave drag with the airfoil shape. However, in this case, the optimizer is unable to produce the heavily aft loaded airfoils that are optimal for this case because they lead to an unstable design. As a result, the optimizer is forced to compromise between reducing the drag and satisfying the static margin constraint. These compromises show up in different ways at the different sections of the wing. At the wing root, the $C_{p}$ profile is spread over the entire chord of the section with significant lift generated at both the leading and trailing edges with relatively little lift generated mid chord. The mid-wing section exhibits a rooftop $C_{p}$ distribution over the front half of the foil, helping to reduce the wave drag of the wing. However, the addition of the static margin constraint prevents the optimizer from extending this trend over the entire foil. As a result, there is a fairly constant amount of lift generated over the forward two thirds of the foil with negative lift generated at the trailing edge. The optimizer has also added more sweep to the wing than in the comparable bending constrained case. As discussed earlier, this reduces the effective Mach number the wing sees and allows tip washout to contribute to wing trim.

In addition to this general discussion, there are two specific secondary characteristics that are worth highlighting. First, the optimizer is adding a significant loading to the bottom surface of the leading edge of the wing at the root. This forward loading helps reduce the moment of the root section. Also, the optimizer has developed an interesting inflection in the camber of the mid-wing airfoil. At the trailing edge, the airfoil starts to develop the high camber shape typical of transonic airfoils, but partway to the trailing edge the foil develops reflex to help reduce the moment of the wing.

\section{Dynamic Stability Constrained Problems}

The CAP constrained optimizations add the dynamic stability constraints to the problem. This requires the consideration of extra stability derivatives, as well as the mass moment of inertia of the aircraft during the optimization. The optimal solution for the CAP constrained planform-only optimization at Mach $=0.5$ - shown in Figure 24 - is essentially the same as the static margin result from Figure 19. This results from the fact that the static margin constraint is still active and that moment of inertia multiplier is able to raise the moment of inertia sufficiently to satisfy the CAP 


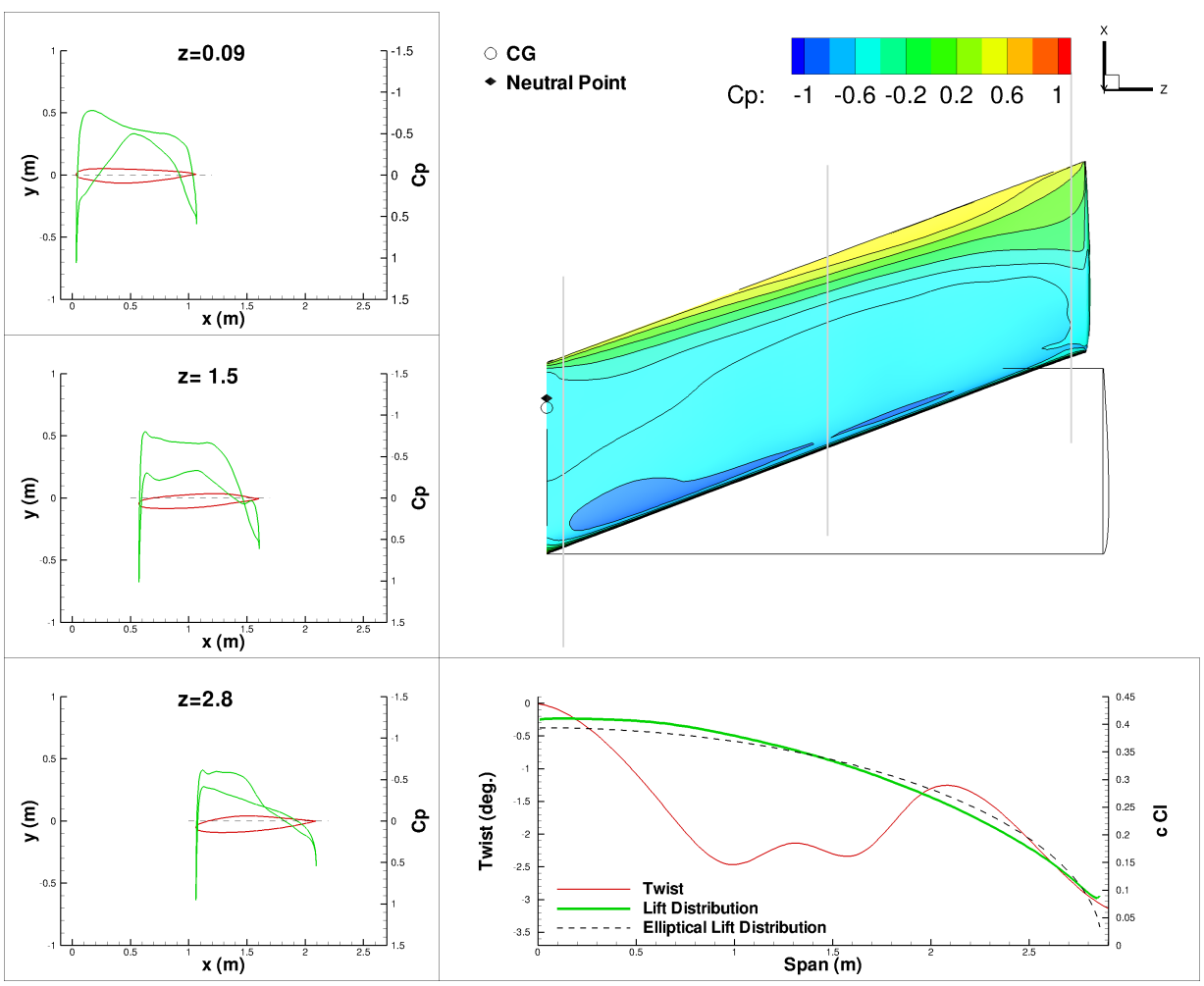

Figure 23. $K_{n}$ constrained optimization: planform and shape variables: $\mathbf{M = 0 . 8 5}$

and damping constraints. If the moment of inertia multiplier were limited to a smaller value, the optimal solution would likely be more highly swept with a larger static margin.

An interesting result from the addition of the dynamic constraints is that the addition of the shape variables no longer produces an unswept wing. As shown in Figure 25, the optimal solution now has almost 20 degrees of sweep. This results largely from the need to maintain a high enough moment of inertia to satisfy the damping requirements. Another interesting side effect of this result is that the section profiles no longer exhibit any reflex at the trailing edge. This comes from the fact that the optimizer is now able to use sweep and tip washout to trim the aircraft without any additional penalty, so the $C_{p}$ distribution is not required to add reflex to do so.

The planform-only, Mach $=0.7, \mathrm{CAP}$ constrained case produces a result similar to static margin constrained case. As with the Mach $=0.5$ case, the moment of inertia multiplier is able to increase the moment of inertia to the point where the CAP constraint and damping constraints are satisfied.

As with the previous CAP constraint cases, at Mach $=0.7$ the shape variables are not sufficient to allow the optimizer to reduce the sweep of the wing to zero. Again the optimal results from the bending constrained case and the static-stability constrained cases have insufficient damping to satisfy the dynamic constraints. In this case, however, the added sweep causes the static margin constraint to be inactive, though the mid-span section still develops a significant amount of reflex.

At Mach $=0.85$ the CAP constrained result is essentially the same as the static margin constrained result. Because the shape variables were not able to completely eliminate the sweep at this higher Mach number, the static margin case has enough sweep to ensure dynamic stability given a sufficient moment of inertia. Thus, the optimal result exhibits the same combinations of $C_{p}$ distributions and planform variables as the static margin constrained case.

\section{Results Tables}

To compare the results in a more quantitative fashion, the results of the optimizations are presented in tabular form below. For the simpler cases where the various stability parameters were not included in the optimizations, the remaining parameters have been calculated for comparison purposes. A short summary of the most significant parameters is shown in Tables 5 through 9 to support the following discussion. Note that the drag values presented are from steady-state simulations of the optimal shapes for each case. The remainder of the values are computed using the 


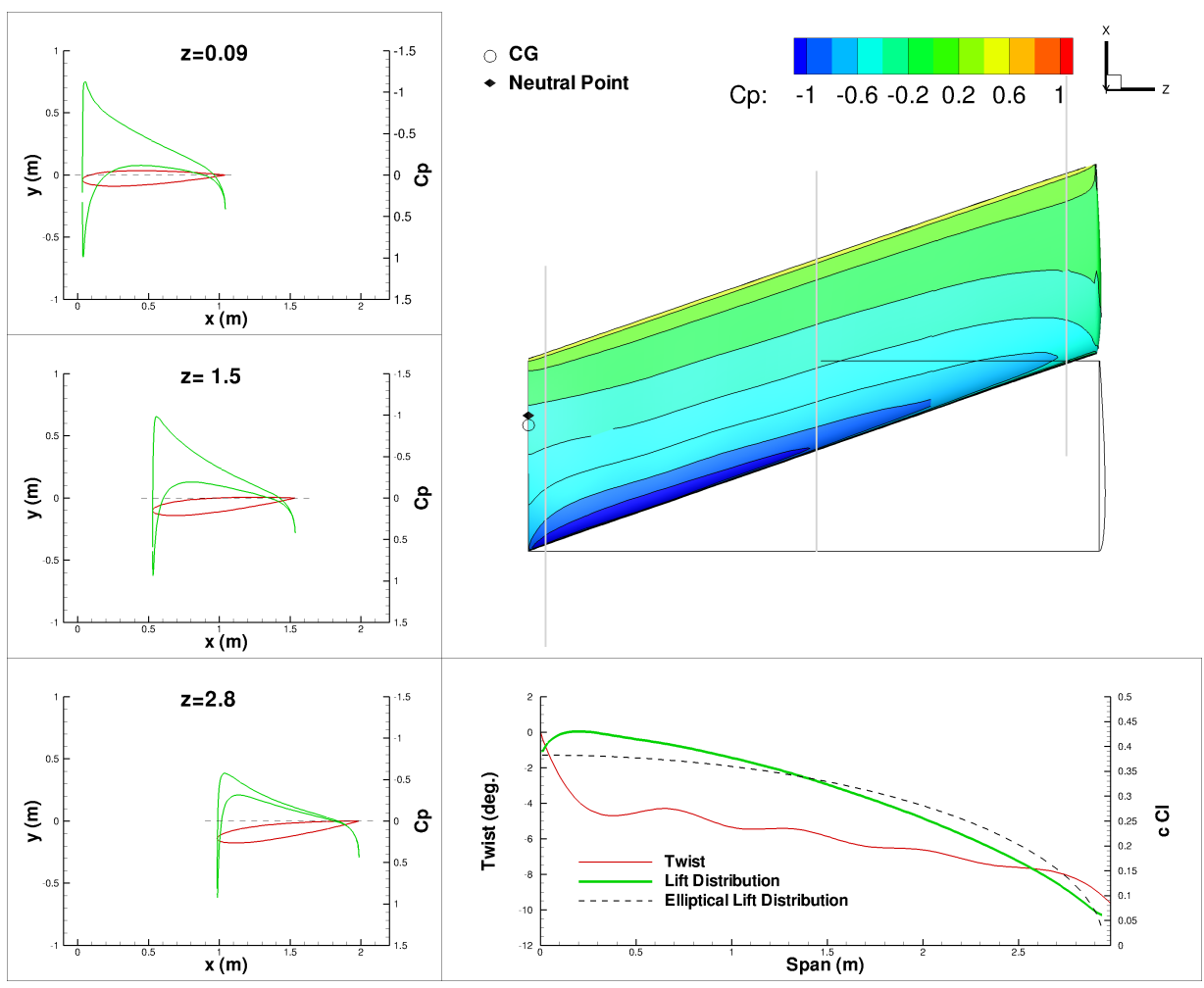

Figure 24. CAP constrained optimization: planform variables only: $\mathbf{M = 0 . 5}, e=0.942$

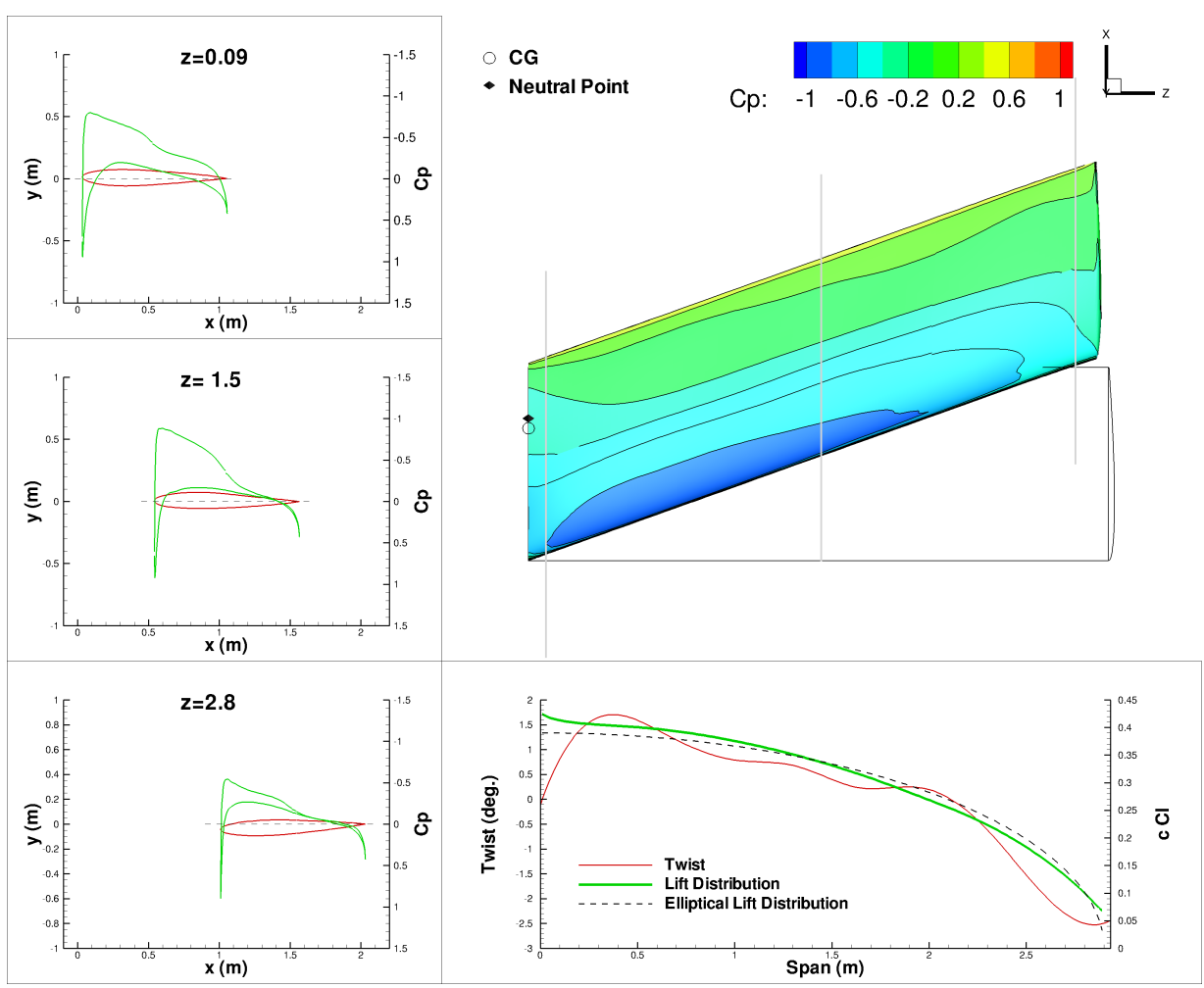

Figure 25. CAP constrained optimization: planform and shape variables: $\mathbf{M}=\mathbf{0 . 5}, e=0.970$ 


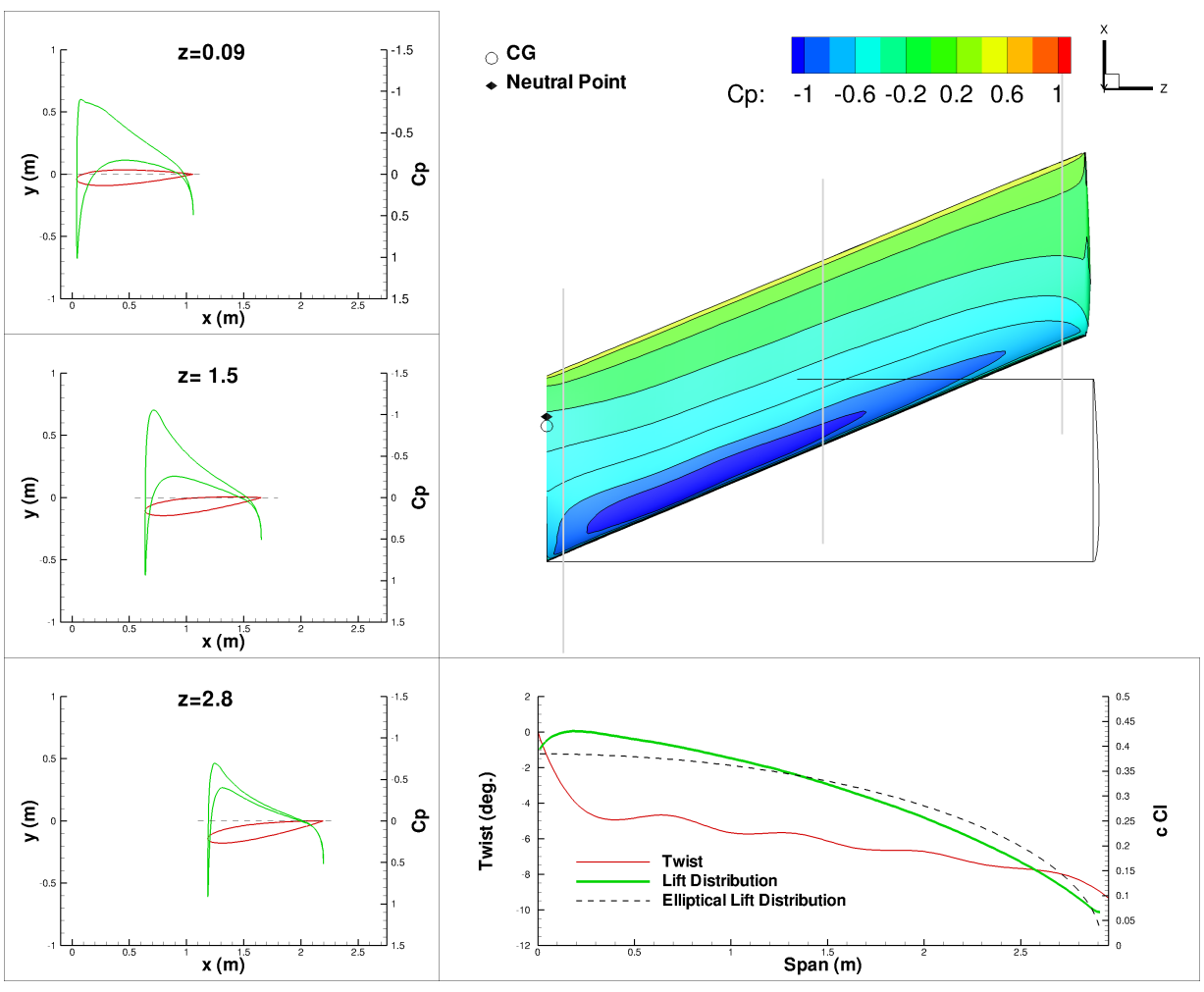

Figure 26. CAP constrained optimization: planform variables only: $M=0.7$

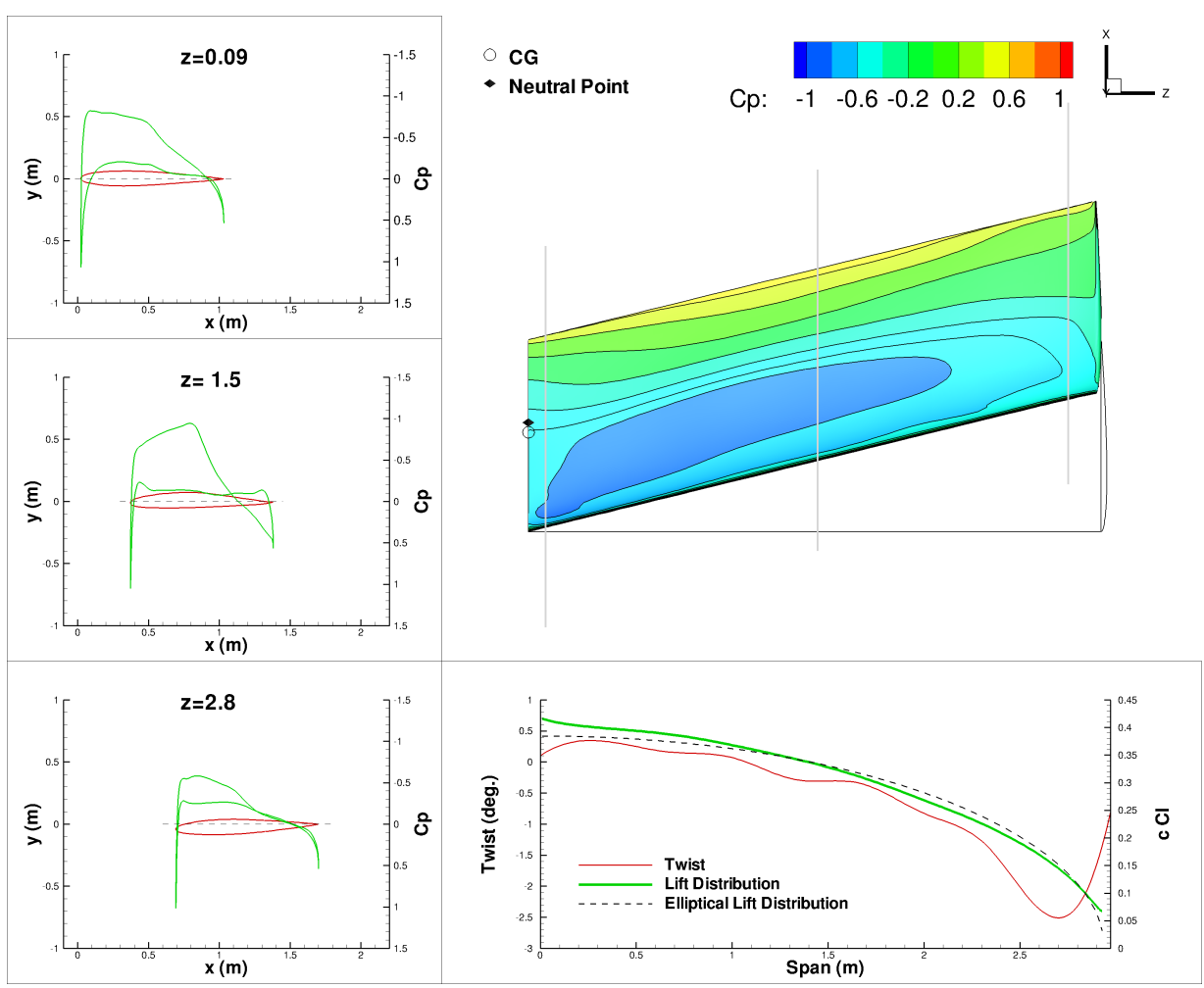

Figure 27. CAP constrained optimization: planform and shape variables: $M=0.7$ 


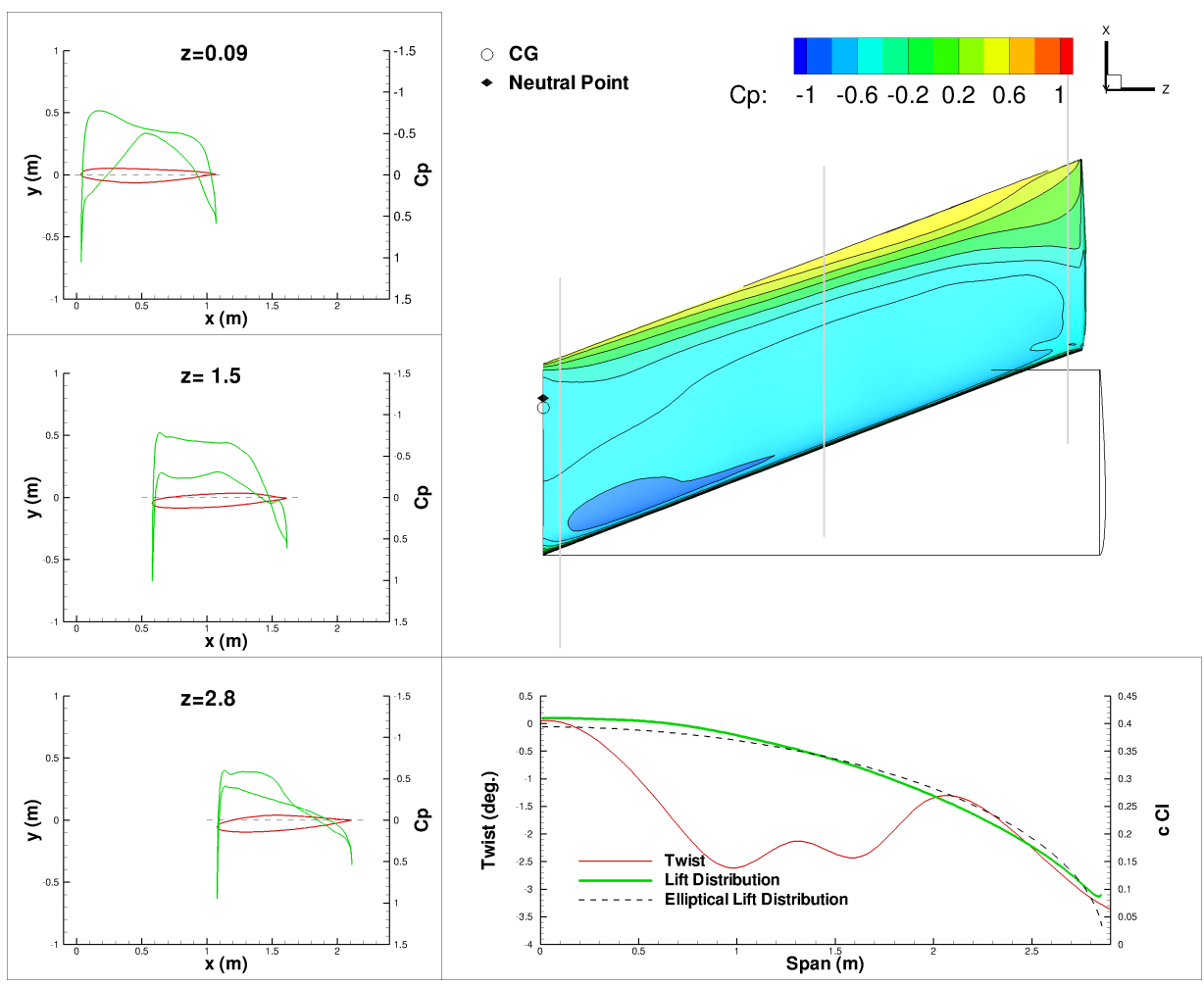

Figure 28. CAP constrained optimization: planform and shape variables: $M=0.85$

time-spectral methods used in the optimizations.

\section{A. $\quad$ Mach $=0.5$ Cases}

The key results for the Mach $=0.5$ cases are shown in Tables 5 and 6 . The first parameter to consider is the drag for each of these cases. The drag for the planform-only cases - shown in Table 5 - show precisely what one would expect. The optimal elliptical solution from the twist-only optimization has a lower drag than the baseline case. The predicted value of drag for this case is 0.00489 , within $2.5 \%$ of the theoretical value of 0.00477 , leading to a span efficiency of 0.977 . The remaining error in this case can be attributed to numerical errors - artificial dissipation for example - as well as the three-dimensional flow at the wing tip.

The added flexibility of the span variable, introduced in the bending moment constrained case, allows the optimizer to further reduce the drag in the baseline aerostructural case. Considering the problem from this perspective allows an additional $1.4 \%$ reduction in drag. Once the stability constraints are included in the optimization, the drag increases. This increase in drag comes from the changes necessary to make the aircraft stable. For the static margin and CAP constrained cases, the necessary increase in sweep is significant, causing a significant increase in the drag.

Also of note are the span efficiency values for the various optimal solutions. In each case, the span efficiency is lower than for the elliptic solution. This is unsurprising, as the lift distributions in these case deviate from elliptical. While the span efficiency is lower for the bending moment constrained case, the increased aspect ratio of this design makes up for the loss of span efficiency, allowing for a slightly lower overall drag coefficient. In the case of the static margin and CAP constrained cases, both the aspect ratio and the span efficiency are lower than in the elliptical case, leading to a higher drag coefficient.

Looking at the values of the various stability constraints, the results show that the static-stability constraint is active in each of the stability constrained cases. Thus, the desired static margin is achieved in both cases. Also note that the three cases without the stability constraints are statically unstable. Finally, the CAP constrained case is essentially identical to the static margin constrained case. This is primarily because the inertia modifier is able to increase the moment of inertia sufficiently to satisfy the damping constraint without modifying the geometry.

The results for the shape optimization cases in Table 6 show a very different picture. In these cases, the staticstability constrained case has essentially the same drag as the planform-only bending constrained case. This comes from the fact that both the aspect ratio and span efficiency are similar to the planform-only bending constrained case. 


\begin{tabular}{lrrrrr}
\hline \hline Parameter & Baseline & Elliptic & Bending & $K_{n}$ & CAP \\
\hline$C_{D}$ & 0.004954 & 0.004888 & 0.004817 & 0.005123 & 0.005133 \\
$C_{m_{\alpha}}$ & 0.002242 & 0.013867 & 0.029788 & -0.246456 & -0.246417 \\
\hline$K_{n}(\%)$ & -0.044805 & -0.277744 & -0.595908 & 4.999788 & 5.000118 \\
$e(\%)$ & 0.963761 & 0.976806 & 0.963537 & 0.943660 & 0.941934 \\
CAP & 0.000258 & -0.015125 & -0.037377 & 0.167942 & 0.168177 \\
$\zeta_{s p}$ & 8.047202 & -0.000000 & -0.000000 & 0.301925 & 0.301636 \\
$\omega_{n}(\mathrm{rad} / \mathrm{s})$ & 0.065557 & -0.000000 & -0.000000 & 1.651115 & 1.652217 \\
$I_{z z}\left(\mathrm{~kg} m^{2}\right)$ & 244.664280 & 242.153023 & 228.835160 & 494.760972 & 493.932604 \\
\hline$\alpha(\mathrm{deg})$. & 3.434484 & 4.042761 & 7.545133 & 9.065110 & 8.899279 \\
$\lambda(\mathrm{deg})$. & 0.000000 & 0.000000 & 0.000000 & 19.280100 & 19.250614 \\
$b(\mathrm{~m})$ & 3.000000 & 3.000000 & 3.042917 & 2.981338 & 2.981104 \\
$P_{I_{z z}}$ & 10.000000 & 10.000000 & 10.000000 & 10.000000 & 10.000000 \\
\hline \hline
\end{tabular}

Table 5. NACA 0012 wing: planform only optimization results: $1107 \mathrm{k}$ cells, $M=0.5$

Further, the results show that the static stability constraint is not active for this case. However, the $C_{p}$ distributions from Figures 19 and 21 clearly show that the solution is different for the planform-only cases and the shape optimization cases. This leads to the conclusion that, for subsonic cases, airfoil shape can be used to trim the aircraft for a wide variety of CG locations with little penalty in terms of drag. This supports the idea that a model with more stringent limits on CG could be used in the context of this case and still allow the optimizer to find feasible solutions, a fact that is not guaranteed for the planform-only cases.

The same is not true for the dynamic stability constrained case. The results for this case show that both the damping constraint and the static margin constraint are active. The addition of the dynamic constraint pushes the optimizer to increase the moment of inertia of the aircraft, which causes an increase in sweep of the wing. Because of the root bending moment constraint, a reduction of the span ensues, causing a 7\% increase in drag even though the span efficiency is similar to the two static-stability constrained cases. Note that the moment of inertia multiplier is also at its upper bound. Therefore, an increase in this limit may reduce the required sweep and thus the amount of drag increase.

\begin{tabular}{lrr}
\hline \hline Parameter & $K_{n}$ & CAP \\
\hline$C_{D}$ & 0.004801 & 0.005149 \\
$C_{m_{\alpha}}$ & -0.752988 & -0.246065 \\
\hline$K_{n}(\%)$ & 15.092049 & 5.000230 \\
$e(\%)$ & 0.968178 & 0.970392 \\
$\mathrm{CAP}$ & 0.586254 & 0.164005 \\
$\zeta_{s p}$ & 0.161038 & 0.299986 \\
$\omega_{n}(\mathrm{rad} / \mathrm{s})$ & 3.142237 & 1.621730 \\
$I_{z z}\left(\mathrm{~kg} m^{2}\right)$ & 403.325544 & 517.368619 \\
\hline$\alpha($ deg. $)$ & 3.057671 & 3.051991 \\
$\lambda($ deg. $)$ & 0.000000 & 19.888040 \\
$b(\mathrm{~m})$ & 3.040392 & 2.932521 \\
$P_{I_{z z}}$ & 10.000000 & 10.000000 \\
\hline \hline
\end{tabular}

Table 6. NACA 0012 wing: shape optimization results: $1107 \mathrm{k}$ cells, $\mathrm{M}=0.5$

\section{B. $\quad$ Mach $=0.7$ Cases}

The planform-only cases at Mach $=0.7-$ shown in Table $7-$ are not as interesting as the Mach $=0.5$ cases. The optimal bending constrained result has a static margin of roughly $0.7 \%$ and thus is stable. The use of the static margin constraint requires an increase in sweep to provide the necessary static margin. However, this additional sweep, along with a relatively large moment of inertia are sufficient to satisfy the CAP and damping constraints. Thus, the CAP constrained case has essentially the same optimal solution as the static margin constrained case.

Examining the shape optimization cases at Mach $=0.7$ - shown in Table $8-$, the results once again show what one would expect. The bending constrained case produces a drag result that is within one count of the optimal solutions at Mach $=0.5$. This highlights the ability of the shape optimizations to modify the airfoil shapes to eliminate the wave drag caused by transonic flow. While the bending constrained case is statically unstable, the addition of the static-stability constraint does not cause any significant change in the drag. Thus, the optimizer is able to find airfoil 


\begin{tabular}{lrrrr}
\hline \hline Parameter & Baseline & Bending & $K_{n}$ & CAP \\
\hline$C_{D}$ & 0.005570 & 0.005207 & 0.005325 & 0.005325 \\
$C_{m_{\alpha}}$ & 0.087088 & -0.043966 & -0.284135 & -0.284114 \\
\hline$K_{n}(\%)$ & -1.491942 & 0.745596 & 4.999817 & 4.999817 \\
CAP & -0.055895 & 0.020482 & 0.087320 & 0.087312 \\
$\zeta_{s p}$ & -0.000000 & 0.672371 & 0.310233 & 0.310233 \\
$\omega_{n}(\mathrm{rad} / \mathrm{s})$ & -0.000000 & 0.631212 & 1.272602 & 1.272483 \\
$I_{z z}\left(\mathrm{~kg} m^{2}\right)$ & 417.494607 & 623.660078 & 963.347405 & 963.470201 \\
\hline$\alpha($ deg. $)$ & 2.933511 & 7.613268 & 8.590863 & 8.557310 \\
$\lambda($ deg.) & 0.000000 & 14.106302 & 22.861602 & 22.864088 \\
$b(\mathrm{~m})$ & 3.000000 & 2.980515 & 2.953919 & 2.953687 \\
$P_{I_{z z}}$ & 16.184566 & 16.184566 & 16.184566 & 16.184566 \\
\hline \hline
\end{tabular}

Table 7. NACA 0012 wing: planform only optimization results: $1107 \mathrm{k}$ cells, $M=0.7$

profiles that are able to both eliminate the transonic wave drag and satisfy the moment criteria necessary for staticstability at the same time. As with the Mach $=0.5$ case, there is enough flexibility in the airfoil shape to allow a wide variety of CG locations.

As with the Mach $=0.5$ optimization cases, the CAP constraint forces the optimizer to add sweep to the wing to increase the damping ratio of the aircraft. As a result, there is a corresponding increase in the amount of drag produced by the wing. Note also that the inertia modifier is double what it was for the Mach $=0.5$ case.

\begin{tabular}{lrrr}
\hline \hline Parameter & Bending & $K_{n}$ & CAP \\
\hline$C_{D}$ & 0.004973 & 0.004939 & 0.005090 \\
$C_{m_{\alpha}}$ & 0.539667 & -0.298319 & -0.302163 \\
\hline$K_{n}(\%)$ & -9.112122 & 4.999844 & 5.121750 \\
$\mathrm{CAP}$ & -0.378485 & 0.129099 & 0.099087 \\
$\zeta_{s p}$ & -0.000000 & 0.277583 & 0.299995 \\
$\omega_{n}(\mathrm{rad} / \mathrm{s})$ & -0.000000 & 1.609959 & 1.389344 \\
$\left.I_{z z}(\mathrm{~kg} \mathrm{~m})^{2}\right)$ & 380.807451 & 611.432529 & 847.763119 \\
\hline$\alpha($ deg. $)$ & 2.987315 & 3.008049 & 3.282016 \\
$\lambda($ deg. $)$ & 0.000000 & 0.529970 & 13.921081 \\
$b(\mathrm{~m})$ & 3.010364 & 3.029077 & 2.972135 \\
$P_{I_{z z}}$ & 20.000000 & 20.000000 & 20.000000 \\
\hline \hline
\end{tabular}

Table 8. NACA 0012 wing: shape optimization results: $1107 \mathrm{k}$ cells, $\mathrm{M}=0.7$

\section{Mach $=0.85$ Cases}

The Mach $=0.85$ results, shown in Table 9, exhibit some interesting characteristics. The bending constrained optimal solution is massively unstable, with a static margin of $-23.5 \%$. The addition of the static stability constraint remedies this deficiency, producing an optimal solution at the limiting value of the constraint. However, in this case the drag does not increase as the additional stability constraints are added. In fact, the most constrained case produces the solution with the lowest drag. This might be explained by the larger spans obtained in the $K_{n}$ and CAP optimal solutions. Finally, as with some of the previous cases, the moment of inertia multiplier is sufficient to satisfy the CAP and damping constraints based on the static margin constrained optimal solution, therefore the static margin constrained optimum and the CAP constrained optimum are essentially the same.

\section{Conclusions}

In this work, we explored the effects of static and dynamic stability constraints on the optimal shape of flying wings. The results showed that stability constraints have a significant impact on the optimal shape of the wing, usually causing a corresponding increase in drag. For cases in the subsonic regime and the lower end of the transonic regime, the study showed that airfoil shape can be used to satisfy the static-stability constraints without significant degradation in performance. For these flow regimes, our results showed that using airfoil shape is preferrable to using wing sweep and twist to satisfy the static-stability requirements. At higher transonic Mach numbers, the degradation in performance is unavoidable and the addition of sweep is necessary, regardless of the airfoil shape, to achieve satisfactory results. The same can not be said for the dynamic stability constrained cases. In these cases, the study 


\begin{tabular}{lrrrr}
\hline \hline Parameter & Baseline & Bending & $K_{n}$ & CAP \\
\hline$C_{D}$ & 0.039898 & 0.005792 & 0.005640 & 0.005619 \\
$C_{m_{\alpha}}$ & 0.055392 & 2.566048 & -0.484800 & -0.489347 \\
\hline$K_{n}(\%)$ & -0.729501 & -23.522592 & 4.999816 & 5.051372 \\
$\mathrm{CAP}$ & -0.036492 & -0.975527 & 0.086524 & 0.086135 \\
$\zeta_{s p}$ & -0.000000 & -0.000000 & 0.308502 & 0.309052 \\
$\omega_{n}(\mathrm{rad} / \mathrm{s})$ & -0.000000 & -0.000000 & 1.644602 & 1.639253 \\
$\left.I_{z z}(\mathrm{~kg} \mathrm{~m})^{2}\right)$ & 316.346371 & 416.989558 & 993.139864 & 1010.020857 \\
\hline$\alpha($ deg. $)$ & 2.169667 & 3.437140 & 3.643078 & 3.665365 \\
$\lambda(\mathrm{deg})$. & 0.000000 & 7.609797 & 20.849705 & 21.144092 \\
$b(\mathrm{~m})$ & 3.000000 & 2.881879 & 2.901532 & 2.898588 \\
$P_{I_{z z}}$ & 21.988981 & 21.988981 & 21.988981 & 21.988981 \\
\hline \hline
\end{tabular}

Table 9. NACA 0012 wing: shape optimization results: $1107 \mathrm{k}$ cells, $M=0.85$

showed that for the subsonic and low transonic cases, sweep was required, regardless of the airfoil shape, in order to raise the damping ratio of the aircraft to acceptable levels. At the higher speed in the transonic regime, the static stability constrained results required sufficient sweep to be feasible for both the static and dynamic stability constraints. Thus, the results of this study underline the importance of considering both static and dynamic stability considerations in the design of flying-wing aircraft.

\section{Acknowledgments}

We would like to thank Edwin van der Weide and Juan J. Alonso for their assistance in various stages of this project. We would also like thank Gaetan Kenway for his contributions to the development of the adjoint solver used in this work. Computations were performed on the GPC supercomputer at the SciNet HPC Consortium. SciNet is funded by: the Canada Foundation for Innovation under the auspices of Compute Canada; the Government of Ontario; Ontario Research Fund - Research Excellence; and the University of Toronto. 


\section{References}

[1] Liebeck, R. H., “Design of the Blended Wing Body Subsonic Transport,” Journal of Aircraft, Vol. 41, 2004 , pp. 10-25.

[2] Wakayama, S. and Kroo, I., "The Challenge and Promise of Blended-Wing-Body Optimization,” AIAA Paper 1998-4736, 1998.

[3] Perez, R. E., Liu, H. H., and Behdinan, K., "Multidisciplinary Optimization Framework for Control-Configuration Integration in Aircraft Conceptual Design," Journal of Aircraft, Vol. 43, No. 6, 2006, pp. 1937-1948.

[4] Jameson, A., “Aerodynamic Design via Control Theory,” Journal of Scientific Computing, Vol. 3, No. 3, sep 1988, pp. 233260.

[5] Reuther, J. J., Jameson, A., Alonso, J. J., , Rimlinger, M. J., and Saunders, D., "Constrained Multipoint Aerodynamic Shape Optimization Using an Adjoint Formulation and Parallel Computers, Part 1," Journal of Aircraft, Vol. 36, No. 1, 1999, pp. 5160.

[6] Reuther, J. J., Jameson, A., Alonso, J. J., , Rimlinger, M. J., and Saunders, D., "Constrained Multipoint Aerodynamic Shape Optimization Using an Adjoint Formulation and Parallel Computers, Part 2," Journal of Aircraft, Vol. 36, No. 1, 1999, pp. 6174.

[7] Hicken, J. E. and Zingg, D. W., "Induced-Drag Minimization of Nonplanar Geometries Based on the Euler Equations," AIAA Journal, Vol. 48, No. 11, 2010, pp. 2564-2575. doi:10.2514/1.J050379.

[8] Mader, C. A. and Martins, J. R. R. A., "Stability-Constrained Aerodynamic Shape Optimization of a Flying Wing Configuration," Proceedings of the $13^{\text {th }}$ AIAA/ISSMO Multidisciplinary Analysis and Optimization Conference, Fort Worth, TX, 2010, AIAA 2010-9199.

[9] van der Weide, E., Kalitzin, G., Schluter, J., and Alonso, J. J., "Unsteady Turbomachinery Computations Using Massively Parallel Platforms," Proceedings of the 44th AIAA Aerospace Sciences Meeting and Exhibit, Reno, NV, 2006, AIAA 20060421.

[10] Mader, C. A. and Martins, J. R. R. A., "Computation of Aircraft Stability Derivatives Using an Automatic Differentiation Adjoint Approach,” AIAA Journal, 2011. doi:10.2514/1.J051147, In Press.

[11] Gopinath, A. K., Efficient Fourier-Based Algorithms for Time-Periodic Unsteady Problems, Ph.D. thesis, Stanford University, Stanford, CA 94305, April 2007.

[12] Perez, R. E., Jansen, P. W., and Martins, J. R. R. A., "pyOpt: A Python-Based Object-Oriented Framework for Nonlinear Constrained Optimization," Structures and Multidisciplinary Optimization, Vol. 45, No. 1, 2012, pp. 101-118. doi:10.1007/s00158-011-0666-3.

[13] Gill, P. E., Murray, W., and Saunders, M. A., "SNOPT: An SQP Algorithm for Large-Scale Constrained Optimization,” SIAM Review, Vol. 47, No. 1, 2005, pp. 99-131.

[14] Perez, R. E. and Martins, J. R., "pyACDT: An Object-Oriented Framework for Aircraft Design Modelling and Multidisciplinary Optimization," Proceedings of the $12^{\text {th }}$ AIAA/ISSMO Multidisciplinary Analysis and Optimization Conference, Victoria, BC, September 2008, AIAA 2008-5955.

[15] Kenway, G. K., Kennedy, G. J., and Martins, J. R. R. A., "A CAD-Free Approach to High-Fidelity Aerostructural Optimization," Proceedings of the $13^{\text {th }}$ AIAA/ISSMO Multidisciplinary Analysis and Optimization Conference, Fort Worth, TX, 2010, AIAA 2010-9231.

[16] Chai, S., Crisafulli, P., and W.H.Mason, "Aircraft Center of Gravity Estimation in Conceptual/Preliminary Design,” Proceedings of the 1st AIAA Aircraft Engineering, Technology, and Operations Congress, Los Angeles, CA, 1995, AIAA 95-3882.

[17] ESDU, "Geometrical Properties of Cranked and Straight Tapered Wing Planforms," Tech. Rep. Item No. 76003, ESDU International plc, London, UK, January 1976.

[18] Murman, S. M., "Reduced-Frequency Approach for Calculating Dynamic Derivatives," AIAA Journal, Vol. 45, No. 6, 2007, pp. 1161-1168.

[19] Ronch, A. D., Ghoreyshi, M., Badcock, K., Görtz, S., Widhalm, M., Dwight, R., and Campobasso, M., "Linear Frequency Domain and Harmonic Balance Predictions of Dynamic Derivatives," Proceedings of the $28^{\text {th }}$ AIAA Applied Aerodynamics Conference, Chicago, IL, 2010, AIAA 2010-4699.

[20] Etkin, B., Dynamics of Atmospheric Flight, Dover Publications, Mineola, New York, 2000.

[21] Agenbag, D. S., Longitudinal Handing Characteristics of a Tailless Gull-Wing Aircraft, Master's thesis, University of Pretoria, Pretoria, South Africa, July 2008.

[22] McRuer, D., Ashkenas, I., and Graham, D., Aircraft Dynamics and Automatic Control, Princeton University Press, Princeton, New Jersey, 1973.

[23] Bihrle, W., “A Handling Qualities Theory for Precise Flight Path Control,” AFFDL Technical Report AFFDL-TR-65-198, 1966. 
[24] Military, U. S., "Flying Qualities of Piloted Airplanes,” Military Specification MIL-F-8785c, 1980.

[25] Cramer, E. J., Dennis, J. E., Frank, P. D., Lewis, R. M., and Shubin, G. R., "Problem Formulation for Multidisciplinary Optimization,” SIAM Journal on Optimization, Vol. 4, No. 4, 1994, pp. 754-776. doi:10.1137/0804044.

[26] Haftka, R. T., "Simultaneous Analysis and Design," AIAA Journal, Vol. 23, No. 7, July 1985, pp. $1099-1103$. doi:10.2514/3.9043.

[27] Braun, R. D. and Kroo, I. M., "Development and Application of the Collaborative Optimization Architecture in a Multidisciplinary Design Environment," Multidisciplinary Design Optimization: State-of-the-Art, edited by N. Alexandrov and M. Y. Hussaini, SIAM, 1997, pp. 98-116.

[28] Bloebaum, C. L., Hajela, P., and Sobieszczanski-Sobieski, J., "Non-Hierarchic System Decomposition in Structural Optimization," Engineering Optimization, Vol. 19, No. 3, 1992, pp. 171-186. doi:10.1080/03052159208941227.

[29] Sobieszczanski-Sobieski, J., Agte, J. S., and Sandusky, R. R., "Bi-Level Integrated System Synthesis," AIAA Journal, Vol. 38, No. 1, January 2000, pp. 164-172. doi:10.2514/2.937.

[30] Kim, H. M., Michelena, N. F., Papalambros, P. Y., and Jian, T., “Target Cascading in Optimal System Design,” Journal of Mechanical Design, Vol. 125, No. 3, September 2003, pp. 474-480. doi:10.1115/1.1582501.

[31] Lambe, A. B. and Martins, J. R. R. A., "Extensions to the Design Structure Matrix for the Description of Multidisciplinary Design, Analysis, and Optimization Processes," Structural and Multidisciplinary Optimization, 2012. doi:10.1007/s00158012-0763-y, (In press). 